\title{
CATEGORIES OF LAYERED SEMIRINGS
}

\author{
ZUR IZHAKIAN, MANFRED KNEBUSCH, AND LOUIS ROWEN
}

\begin{abstract}
We generalize the constructions of [17, 19] to layered semirings, in order to enrich the structure and provide finite examples for applications in arithmetic (including finite examples). The layered category theory of [19] is extended accordingly, to cover noncancellative monoids.
\end{abstract}

\section{INTRODUCTION}

This paper is a continuation of [17] and [19. Tropical mathematics often involves the study of valuations, whose targets are ordered Abelian groups that can be viewed as max-plus algebras. The layered supertropical domain was introduced in [17, and put in a categorical framework in [19, in order to provide algebraic tools with which to study this structure.

Tropical mathematics often involves the study of valuations, whose targets are ordered Abelian groups that can be viewed as max-plus algebras. The basic functor used in [19] goes from the category of cancellative ordered Abelian monoids to the category of $L$-layered domains ${ }^{\dagger}$ with respect to a semiring ${ }^{\dagger} L$. (We use the generic notation ${ }^{\dagger}$ to indicate that we do not require a zero element.)

On the other hand, many important classical arithmetical results are proved by passing to finite structures (i.e., modulo a prime number). The main objective of this paper is to open the way to an arithmetic tropical theory, by permitting finite tropical structures. This might seem to be an oxymoron, since all nontrivial ordered groups are infinite. But valuation theory has been enriched in [11] and [31] by means of valuations to arbitrary ordered Abelian monoids, thereby raising the possibility of a layered semiring $^{\dagger}$ construction for any ordered Abelian monoid.

Since any cancellative ordered monoid is necessarily infinite, we need to include noncancellative monoids in our category if we want to deal with finite structures and their corresponding arithmetic. But then, as observed already in [17, the naive analog of [17, Construction 3.2] does not satisfy distributivity, so we must turn to a more sophisticated version, given below in Construction 3.5. This requires a 0-layer, i.e., $0 \in L$, at the cost of a decidedly more complicated multiplication. So at the outset we consider 'absorption' via the elements $\mathbb{0}$ and $\infty$.

The dividend is far greater flexibility in our examples, cast in a more general categorical setting than given in [19. Construction [3.5 is verified in Theorem 3.6. In the process we obtain finite structures, as indicated in Example 4.19. Namely, applying "truncation" both to the given valued monoid and the sorting set yields finite examples and could permit one to apply the corresponding arithmetic tools. Since the 0 layer and infinite layer both play significant roles in this theory, we study their properties and interactions in detail in 4

Several serious technical difficulties arise when we try to put this more general construction in its categorical context, because a homomorphism of monoids might send a noncancellative monoid to a cancellative monoid, thereby requiring us to switch back and forth from one construction to the other.

Date: July 10, 2018.

2010 Mathematics Subject Classification. Primary 06F20, 11C08, 12K10, 14T05, 14T99, 16Y60; Secondary 06F25, $16 \mathrm{D} 25$.

Key words and phrases. Tropical categories, tropical algebra, tropical geometry, valued monoids, valuations, tropicalization, root sets, component sets.

This research of the first and third authors is supported by the Israel Science Foundation (grant No. 448/09).

This research of the first author has been supported by the Oberwolfach Leibniz Fellows Programme (OWLF), Mathematisches Forschungsinstitut Oberwolfach, Germany.

The second author was supported in part by the Gelbart Institute at Bar-Ilan University, the Minerva Foundation at Tel-Aviv University, the Mathematics Dept. of Bar-Ilan University, and the Emmy Noether Institute. 
The corresponding maps apparently cannot be written as morphisms of semirings ${ }^{\dagger}$, so one must broaden either the class of morphisms or the class of objects in the category.

We try both approaches in turn, the first approach occupying the body of this paper and the second approach discussed in the appendix. In $\$ 4$ we introduce our main examples. In \$5, which still is not cast in full generality, we pass from the category of valued monoids to the category of semirings ${ }^{\dagger}$ by means of the "0-excepted" homomorphisms of Definition 5.14. This permits us in 86 to describe the tropicalization functor more generally, for rings that need not be integral domains. Furthermore, if one turns to the basic link of tropical geometry with classical algebraic geometry via valuations, one is led to consider more general "transmissions" which pass from valuation to valuation.

The key to tying this in with tropicalization is Kapranov's Lemma. Elaborating on [19, §8], we show in Remark 6.6 how Kapranov's Lemma can be expressed in terms of a Kapranov map, thereby yielding a "layering" functor for polynomial functions. This map is compatible with the tropicalization map given in 27 .

In 97 we see that the category LaySemi ${ }^{\dagger}$ of layered semirings ties in to layered supervaluations, and specializes to the category STROP from [20], when we take $L=\{0,1, \infty\}$ and $R_{0}=\left\{\mathbb{D}_{R}\right\}$. The key result in this regard is Theorem 7.8 and its corollary, which show that the transmissions of layered supervaluations often become layered homomorphisms under certain natural assumptions.

In Appendix A (8) even fuller generality is obtained by considering structures more general than semirings $^{\dagger}$, analogous to the supertropical monoids of [20]. Here the noncancellative products belong to the 0-layer, for which addition with the rest of the structure is not defined; as a result, we do not quite have a semiring ${ }^{\dagger}$.

\section{BACKGROUND}

For us, a monoid is a multiplicative semigroup with a unit element $\mathbb{1}_{\mathcal{M}}$. We work with semirings and their (multiplicative) monoids.

2.1. Semigroups and semirings. We review a few definitions from semigroups and semirings. We say that an element $a$ of a semigroup $\mathcal{M}:=(\mathcal{M}, \cdot)$ is partially absorbing if $a b=a$ for some $b \in \mathcal{M}$; an element $a$ of $\mathcal{M}:=(\mathcal{M}, \cdot)$ is absorbing if $a b=b a=a$ for all $b \in \mathcal{M}$.

Lemma 2.1. If $\mathcal{M}$ is an Abelian semigroup with a unique partially absorbing element a, then $a$ is absorbing.

Proof. Suppose $a b=a$. For any $c \in \mathcal{M}$ we have $(c a) b=c(a b)=c a=a c$. Thus, $a c$ is partially absorbing, implying $a c=a$ by hypothesis.

Usually, the absorbing element is identified with $\mathbb{O}$, but it could also be identified with the infinite element $\infty$, given by

$$
\infty \cdot a=a \cdot \infty=\infty, \quad \text { for all } 0 \neq a \in \mathcal{M} .
$$

(We do not necessarily assume that $\mathcal{M}$ contains $\mathbb{0}$ or $\infty$. The partially absorbing element $\infty$ is absorbing when $\mathbb{0} \notin \mathcal{M}$.)

A semigroup $\mathcal{M}$ is pointed if it has an absorbing element $\mathbb{D}_{\mathcal{M}}$.

A semigroup $\mathcal{M}$ is cancellative with respect to a subset $S$ if $a s=b s$ implies $a=b$ whenever $a, b \in \mathcal{M}$ and $s \in S$. A pointed semigroup $\mathcal{M}$ is cancellative if $\mathcal{M}$ is cancellative with respect to $\mathcal{M} \backslash\left\{\mathbb{D}_{\mathcal{M}}\right\}$.

An element $\infty$ in a semiring ${ }^{\dagger} R$ is infinite if it is absorbing with respect to addition, i.e., satisfies

$$
r+a=r \quad \text { for all } a \in R .
$$

Definition 2.2. A domain ${ }^{\dagger}$ is a semiring ${ }^{\dagger} R$ that is cancellative under multiplication. A semiring $R$ is a domain if $R \cup\{\mathbb{O}\}$ is a domain ${ }^{\dagger}$. Likewise, $R$ is a semifield ${ }^{\dagger}$ if $R$ is closed under multiplication. $R$ is a semifield if $R \cup\{\mathbb{O}\}$ is a semifield ${ }^{\dagger}$.

Although we have two usages for 'infinite,' one additive and one multiplicative, they are connected by the following observation:

Proposition 2.3. If $R_{\infty}=R \cup\{\infty\}$ where $R$ is a semifield ${ }^{\dagger}$ and $\infty \in R_{\infty}$ is an infinite element in the sense of (2.2), then $a:=\infty$ also satisfies (2.1). 
Proof. $a=a+a b=\left(a b^{-1}+a\right) b=a b$.

Congruences over semifields ${ }^{\dagger}$ are described in detail in 13. (The domains ${ }^{\dagger}$ of eventual tropical interest to us are polynomial semirings ${ }^{\dagger}$ over semifields ${ }^{\dagger}$, which are needed to define tropical varieties, as described in [17, 19.

As in [19] we work with the category $\operatorname{Semir}^{\dagger}$ of semirings ${ }^{\dagger}$ and their homomorphisms, as compared to the category Semir of semirings and semiring homomorphisms. We refer the reader to 19 for preliminary facts that we need; an earlier reference is [5. As noted in [19, the category Semir ${ }^{\dagger}$ is isomorphic to a subcategory of the category Semir, since any semiring ${ }^{\dagger} R$ can be embedded in a semiring $R \cup\{\mathbb{D}\}$ by formally adjoining a zero element $\mathbb{D}$.

2.1.1. Pre-ordered semigroups and semirings ${ }^{\dagger}$.

Definition 2.4. A semigroup $\mathcal{M}:=(\mathcal{M}, \cdot)$ (or a monoid $\mathcal{M}:=\left(\mathcal{M}, \cdot, \mathbb{1}_{\mathcal{M}}\right)$ ) is pre-ordered (resp. partially pre-ordered, partially ordered, ordered) if it has a pre-order $\leq$ (resp. partially pre-order, partial order, order) such that

$$
b \leq c \text { implies } a b \leq a c \text { and } b a \leq c a, \quad \forall a \in \mathcal{M} .
$$

As in 19, we assume that all preorders are positive. PPreOMon, PreOMon, POMon, OMon, and $\mathrm{OMon}^{+}$denote the respective categories of partially pre-ordered, pre-ordered, partially ordered, ordered, and cancellative ordered monoids, whose morphisms are the order-preserving homomorphisms.

The crucial observation here is that any semiring ${ }^{\dagger}$ becomes a partially pre-ordered semigroup via the rule (also cf. [13):

$$
a \leq b \quad \text { iff } \quad a=b \quad \text { or } \quad b=a+c \quad \text { for some } c \in R .
$$

We say that a semiring ${ }^{\dagger} R$ is pre-ordered (resp. partially pre-ordered, partially ordered, ordered) if it has a partial pre-order $\leq$ (resp. partial order, order) with respect to which both the monoid $\left(R, \cdot, \mathbb{1}_{R}\right)$ and the semigroup $(R,+)$ satisfy Condition (2.3) of Definition 2.4.

By [19, Proposition 3.9], there is a natural functor Semir ${ }^{\dagger} \rightarrow$ PPreOMon, where we define the partial pre-order on a semiring ${ }^{\dagger} R$ as in (2.4).

2.2. Valued monoids. Although we focused on ordered monoids in 19, tropical mathematics is concerned with valuations. More generally, we can take the target to be a monoid, cf. [16, Definition 2.1].

Definition 2.5. A monoid $\mathcal{M}:=\left(\mathcal{M}, \cdot, \mathbb{1}_{\mathcal{M}}\right)$ is m-valued with respect to an ordered monoid $\mathcal{G}:=$ $\left(\mathcal{G}, \cdot, \geq, \mathbb{1}_{\mathcal{G}}\right)$ if there is an onto monoid homomorphism $v: \mathcal{M} \rightarrow \mathcal{G}$. (In other words, $v(a b)=v(a) v(b)$.) We also call $v$ an $m$-valuation. We notate this set-up as the triple $(\mathcal{M}, \mathcal{G}, v)$.

This fits in better with our algebraic notation for semirings ${ }^{\dagger}$. Thus, any valuation $v: K \rightarrow \mathcal{G}$ is an $\mathrm{m}$-valuation, where we just disregard addition in $K$. The hypothesis that $v$ is onto can always be attained by replacing $\mathcal{G}$ by $v(\mathcal{M})$ if necessary.

The category of triples should be quite intricate, since the morphisms should include all maps which "transmit" one m-valuation to another. We explore this idea further in 97 but for the most part take a simpler approach, following [19].

Definition 2.6. ValMon is the category of valued monoids whose objects are triples $(\mathcal{M}, \mathcal{G}, v)$ as in Definition 2.5, for which a morphism

$$
\phi:(\mathcal{M}, \mathcal{G}, v) \longrightarrow\left(\mathcal{M}^{\prime}, \mathcal{G}^{\prime}, v^{\prime}\right)
$$

is comprised of a pair $\left(\phi_{\mathcal{M}}, \phi_{\mathcal{G}}\right)$ of a monoid homomorphism $\phi_{\mathcal{M}}: \mathcal{M} \rightarrow \mathcal{M}^{\prime}$, as well as an orderpreserving monoid homomorphism $\phi_{\mathcal{G}}: \mathcal{G} \rightarrow \mathcal{G}^{\prime}$, satisfying the compatibility condition

$$
v^{\prime}\left(\phi_{\mathcal{M}}(a)\right)=\phi_{\mathcal{G}}(v(a)), \quad \forall a \in \mathcal{M} .
$$

Remark 2.7. When the value map $v$ of the triple $(\mathcal{M}, \mathcal{G}, v)$ is 1:1, then $\mathcal{M}$ inherits the order from $\mathcal{G}$, by stipulating that $a<b$ when $v(a)<v(b)$. In this way, we can view OMon as a full subcategory of ValMon. 
2.3. Congruences. Since we work in the framework of universal algebras, we need some general observations, and then specialize to the cases of interest to us (semigroups and semirings). One defines a congruence $\Omega$ of an algebraic structure $\mathcal{A}$ to be an equivalence relation $\equiv$ which preserves all the relevant operations and relations; we call $\equiv$ the underlying equivalence of $\Omega$. Equivalently, a congruence $\Omega$ is a sub-structure of $\mathcal{A} \times \mathcal{A}$ that contains the diagonal $\operatorname{diag}(R):=\{(a, a): a \in R\}$, as described in Jacobson [23, §2].

Since the most important semirings ${ }^{\dagger}$ for us are domains ${ }^{\dagger}$, we want to know, given a congruence $\Omega$ on $R$, when the factor semiring ${ }^{\dagger} R / \Omega$ has an absorbing element, and when it is a domain ${ }^{\dagger}$. Given a subset $A \subset R$, we write $b \equiv A$ if $b \equiv a$ for some $a \in A$. We call an ideal $\mathfrak{a} \triangleleft R$ closed under $\Omega$ if $b \equiv \mathfrak{a}$ implies $b \in \mathfrak{a}$.

Lemma 2.8. Suppose $\Omega$ is a congruence on a semiring ${ }^{\dagger} R$.

(i) $R / \Omega$ is a domain ${ }^{\dagger}$ iff its underlying equivalence $\equiv$ is cancellative, in the sense that $a b \equiv a c$ implies $b \equiv c$.

(ii) If $R / \Omega$ is a semiring with absorbing element, which we denote as $\overline{\mathbb{0}}$, then the pre-image $I$ of $\overline{\mathbb{0}}$ is a closed ideal of $R$ all of whose elements are equivalent. Conversely, if $\mathfrak{a}$ is a closed ideal of $R$ all of whose elements are equivalent, then the image of $\mathfrak{a}$ is the absorbing element of $R / \Omega$.

(iii) When (ii) holds, $R / \Omega$ is a domain iff $\equiv$ is cancellative with respect to all elements not in $\mathfrak{a}$, in the sense that if $a b \equiv$ ac for $a \notin \mathfrak{a}$, then $b \equiv c$.

Proof. Write $\bar{a}$ for the image of $a$ in $R / \Omega$.

(i) $a b \equiv a c$ iff $\bar{a} \bar{b}=\bar{a} \bar{c}$, iff $\bar{b}=\bar{c}$, iff $b \equiv c$.

(ii) If $a, b \in I$, then $\bar{a}=\bar{b}=\overline{\mathbb{0}}$, implying $a \equiv b$. Conversely, if $\mathfrak{a}$ is a closed ideal of $R$ all of whose elements are equivalent, then the image of $\mathfrak{a}$ is an ideal of $R / \Omega$ consisting of a single element, which must thus be the absorbing element.

(iii) The condition translates to saying that $\bar{a} \bar{b}=\bar{a} \bar{c}$ for $\bar{a} \neq \overline{\mathbb{D}}$ implies $\bar{b}=c$.

It is useful to weaken the notion of congruence.

Definition 2.9. A half-congruence $\Omega$ is a sub-structure of $\mathcal{A} \times \mathcal{A}$ that contains the diagonal and is transitive in the sense that if $\Omega$ contains $(a, b)$ and $(b, c)$ then it also contains $(a, c)$.

Throughout the body of this paper $R$ denotes a commutative semiring ${ }^{\dagger}$.

Example 2.10. In the language of monoids, if $\mathfrak{a}_{1}, \mathfrak{a}_{2}$ are monoid ideals of a monoid $\mathcal{M}:=(\mathcal{M}, \cdot)$, then

$$
\left(\mathfrak{a}_{1} \times \mathfrak{a}_{2}\right) \cup\{(a, a): a \in \mathcal{M}\}
$$

is a congruence since $\mathfrak{a}_{i} a \subseteq \mathfrak{a}_{i}$. But in the language of semirings ${ }^{\dagger}$, if $\mathfrak{a}_{1}, \mathfrak{a}_{2}$ are semiring ${ }^{\dagger}$ ideals of a semiring ${ }^{\dagger} R$, then $\left(\mathfrak{a}_{1} \times \mathfrak{a}_{2}\right) \cup\{(r, r): r \in R\}$ need not even be a half-congruence, since it may not be closed under addition. (In general, $\mathfrak{a}_{i}+r \nsubseteq \mathfrak{a}_{i}$.)

Lemma 2.11. A transitive relation $\sim$ is a half-congruence on a semiring if it is closed under addition and multiplication by the diagonal, i.e., if it satisfies the following conditions for all $a_{1}, a_{2}$, and b:

$$
\begin{array}{rlrlrl}
a_{1} & \sim a_{2} & \text { implies } & & a_{1}+b \sim a_{2}+b ; \\
a_{1} \sim a_{2} & \text { implies } & & a_{1} b \sim a_{2} b .
\end{array}
$$

Proof. $a_{1}+b_{1} \sim a_{2}+b_{1}=b_{1}+a_{2} \sim b_{2}+a_{2}=a_{2}+b_{2}$. Likewise for multiplication.

\section{THE LAYERED STRUCTURE}

We are ready to bring in the leading players in this theory, taking into account a 0-layer.

Definition 3.1. A pre-order is directed if for any $a, b$ there is $c$ such that $c \geq a$ and $c \geq b$.

We assume throughout that the sorting set $L$ is a directed, (non-negative) pre-ordered semiring semiring ${ }^{\dagger}$ with zero element $0:=0_{L}$; the bulk of our applications in this paper are for $L$ ordered. Let $L^{\times}:=L \backslash\{0\}$. We recall [17, Construction 3.2]. 
Construction 3.2. Suppose $\mathcal{G}$ is a given cancellative monoid. $R:=\mathscr{R}\left(L^{\times}, \mathcal{G}\right)$ is defined set-theoretically as $L^{\times} \times \mathcal{G}$, where we denote the element $(\ell, a)$ as ${ }^{[\ell]}$ a and, for $k, \ell \in L, a, b \in \mathcal{G}$, we define multiplication componentwise, i.e.,

$$
{ }^{[k]} a \cdot{ }^{[\ell]} b={ }^{[k \ell]} a b .
$$

Addition is given by the rules:

$$
{ }^{[k]} a+{ }^{[\ell]} b= \begin{cases}{ }^{[k]} a & \text { if } a>b, \\ { }^{[\ell]} b & \text { if } a<b, \\ { }^{[k+\ell]} a & \text { if } a=b .\end{cases}
$$

We define $R_{\ell}:=\{\ell\} \times \mathcal{G}$, for each $\ell \in L^{\times}$. Namely $R=\dot{\bigcup}_{\ell \in L} R_{\ell}$.

This is our prototype of a layered pre-domain ${ }^{\dagger}$, and should be borne in mind throughout the sequel. Note that in this case $R_{1}$ is a monoid, which is isomorphic to $\mathcal{G}$.

Nevertheless, we also consider the possibility that the monoid $\mathcal{G}$ is noncancellative, in which case, as noted in [17, Construction 3.2 fails to satisfy distributivity and thus is not a semiring.

Definition 3.3. Suppose $\mathcal{G}$ is an ordered Abelian monoid. An element $z \in \mathcal{G}$ is a noncancellative product if $z=a b=a c$ for suitable $a, b, c$ with $b \neq c$.

More generally, when $(\mathcal{M}, \mathcal{G}, v)$ is a triple, an element $z \in \mathcal{M}$ is a v-noncancellative product if $v(z)=v(a b)=v(a c)$ for suitable $a, b, c$, where $v(b) \neq v(c)$.

Proposition 3.4. The set $A$ of $v$-noncancellative products comprises a monoid ideal of $\mathcal{M}$.

Proof. If $v(z)=v(a b)=v(a c) \in A$, then $v(a d) v(c)=v(a c) v(d)=v(z d)=v(a b d)=v(a d) v(b)$.

Construction 3.5. Suppose $\left(\mathcal{M}, \cdot, \geq, \mathbb{1}_{\mathcal{G}}\right)$ is an Abelian monoid, with an m-valuation $v: \mathcal{M} \rightarrow \mathcal{G}$, and $\mathfrak{a}$ is a monoid ideal of $\mathcal{M}$ containing all v-noncancellative products. $R:=\mathscr{R}(L, \mathcal{M})_{\mathfrak{a}}$ is defined settheoretically as $\left(L^{\times} \times(\mathcal{M} \backslash \mathfrak{a})\right) \cup(\{0\} \times \mathfrak{a})$, where we denote the element $(\ell, a)$ as ${ }^{[\ell]}$ a and, for $k, \ell \in L$, $a, b \in \mathcal{M}$, multiplication is defined componentwise, i.e., via the rules:

$$
{ }^{[k]} a \cdot{ }^{[\ell]} b= \begin{cases}{ }^{[k \ell]} a b & \text { if } a b \notin \mathfrak{a}, \\ { }^{[0]} a b & \text { if } a b \in \mathfrak{a} .\end{cases}
$$

Addition is given as in Construction 3.2

$$
{ }^{[k]} a+{ }^{[\ell]} b= \begin{cases}{ }^{[k]} a & \text { if } v(a)>v(b), \\ { }^{[\ell]} b & \text { if } v(a)<v(b), \\ { }^{[k+\ell]} a & \text { if } v(a)=v(b) .\end{cases}
$$

$R_{0}:=\{0\} \times \mathfrak{a}$ and $R_{\ell}:=\{\ell\} \times(\mathcal{M} \backslash \mathfrak{a})$, for each $\ell \in L^{\times}$. Thus, $R=\dot{\bigcup}_{\ell \in L} R_{\ell}$.

This encompasses the case where $\mathcal{M}=\mathcal{G}$ is an ordered monoid and $v$ is the identity map. We usually refer to this special case, in the interest of clarity.

Theorem 3.6. $R:=\mathscr{R}(L, \mathcal{M})_{\mathfrak{a}}$ is a semiring ${ }^{\dagger}$, while $R$ is a semiring iff the monoid $\mathcal{M}$ is pointed, in which case $\mathbb{O}_{R}={ }^{[0]} \mathbb{O}_{\mathcal{M}}$.

$R \backslash R_{0}$ is a semiring ${ }^{\dagger}$ iff $\mathfrak{a}$ is prime as a monoid ideal of $\mathcal{M}$.

Proof. The verification that $R$ is a semiring ${ }^{\dagger}$ was essentially done in [17, Proposition 3.3]. The trickiest part again is to verify the distributivity law

$$
x(y+z)=x y+x z .
$$

Write $x={ }^{[k]} a, y={ }^{[\ell]} b$, and $z={ }^{[m]} c$, and assume that $v(b) \geq v(c)$. If $v(a b)>v(a c)$, then clearly $v(b)>v(c)$, and

$$
x(y+z)=x y=x y+x z .
$$

Thus we are done unless $v(a b)=v(a c)$.

If $v(b)=v(c)$ with $a b \notin \mathfrak{a}$, then

$$
x(y+z)={ }^{[k]} a\left({ }^{[\ell]} b+{ }^{[m]} b\right)={ }^{[k]} a{ }^{[\ell+m]} b={ }^{[k \ell+k m]}(a b)={ }^{[k \ell]}(a b)+{ }^{[k m]}(a b)=x y+x z .
$$


If $v(b)=v(c)$ with $a b \in \mathfrak{a}$, then

$$
x(y+z)={ }^{[k]} a\left({ }^{[\ell]} b+{ }^{[m]} b\right)={ }^{[k]} a{ }^{[\ell+m]} b={ }^{[0]}(a b)={ }^{[0]}(a b)+{ }^{[0]}(a b)=x y+x z .
$$

If $v(b)>v(c)$, then $a b, a c \in \mathfrak{a}$, so

$$
x(y+z)={ }^{[k]} a\left({ }^{[\ell]} b+{ }^{[m]} c\right)={ }^{[k]} a{ }^{[\ell]} b={ }^{[0]}(a b)={ }^{[0]}(a b)+{ }^{[0]}(a c)=x y+x z .
$$

When $\mathcal{M}$ is pointed, the verification of the zero element is an easy computation.

The next assertion is clear: $x y \in \mathfrak{a}$ iff $x y \in R_{0}$.

We have the maps $\nu_{\ell, k}: R_{k} \rightarrow R_{\ell}$ given by $\nu_{\ell, k}\left({ }^{[k]} a\right)={ }^{[\ell]} a$ for any $0<k \leq \ell$, and a sorting map $\mathrm{s}: R \rightarrow L$ given by $\mathrm{s}(\ell, a)=\ell$, for any $a \in \mathcal{M}, \ell \in L$.

Note that $R \backslash \mathfrak{a}$ could be a finite set, in which case we could apply various arithmetic tools such as zeta functions.

Remark 3.7. If $\mathcal{M}=\mathcal{G}$ and $\mathfrak{a}=\emptyset$, then $R_{0}=\emptyset$, and $\mathscr{R}(L, \mathcal{G})$ coincides with the semiring $\mathscr{R}\left(L^{\times}, \mathcal{G}\right)$ of Construction 3.2.

Lemma 3.8. For any multiplicative idempotent $\ell$ of $L$, the subset $R_{0} \cup R_{\ell}$ of $\mathscr{R}(L, \mathcal{G})$ is a monoid, together with a natural homomorphism to $\mathcal{G}$.

Proof. If ${ }^{[\ell]} a,{ }^{[\ell]} b \in R_{1}$, then their product either is ${ }^{\left[\ell^{2}\right]}(a b)={ }^{[\ell]}(a b) \in R_{\ell}$, or ${ }^{[0]}(a b)$ if $a b \in \mathfrak{a}$. The natural homomorphism is given by ${ }^{[\ell]} a \mapsto v(a)$.

The main application of this lemma is for $\ell=1$. The layer $R_{1}$ is of particular importance, since its unit element is $\mathbb{1}_{R}$. Two other obvious multiplicative idempotents of $L$ are 0 and $\infty$ (when appropriate, since $\infty$ need not belong to $L$ ).

\section{LAYERED SEMIRINGS ${ }^{\dagger}$}

In this section we provide the framework for Construction 3.5 and truncation (Example 4.19). We deal with a zero layer, i.e., assume that $0 \in L$, and treat the zero component $R_{0}$ specially, taking the opportunity to fit the zero element of $R$ (if it exists) into the theory. Since we also want to consider monoids that are not cancellative, we need to work harder to obtain distributivity. We axiomatize in order to place the theory in a categorical framework.

Definition 4.1. Suppose $(L, \geq)$ is a partially pre-ordered, directed semiring. An L-layered semiring ${ }^{\dagger}$

$$
R:=\left(R, L, s,\left(\nu_{m, \ell}\right)\right),
$$

is a semiring ${ }^{\dagger}$, together with a family $\left\{R_{\ell}: \ell \in L\right\}$ of disjoint subsets $R_{\ell} \subset R$, such that

$$
R:=\bigcup_{\ell \in L} R_{\ell}
$$

and a family of sort transition maps

$$
\nu_{m, \ell}: R_{\ell} \rightarrow R_{m}, \quad \forall m \geq \ell>0,
$$

such that

for every $\ell \in L$, and

$$
\nu_{\ell, \ell}=\operatorname{id}_{R_{\ell}}
$$

$$
\nu_{m, \ell} \circ \nu_{\ell, k}=\nu_{m, k}, \quad \forall m \geq \ell \geq k,
$$

whenever both sides are defined. To avoid complications, we assume that any element of $R_{0}$ can be written as a product ab where $a, b \in R \backslash R_{0}$. We also require the axioms $A 1-A 4$, and $B$, given presently, to be satisfied.pave (In order to have our definition compatible with the L-layered pre-domains ${ }^{\dagger}$ of [17, we permit $R_{0}=\emptyset$.)

We also require $R_{\infty}$ to be the direct limit of the $R_{\ell}, \ell>0$, together with maps $\nu_{\infty, \ell}: R_{\ell} \rightarrow R_{\infty}$, which extend to a map $\nu: R \rightarrow R_{\infty}$. (For $c=a b \in R_{0}$ we define $\nu(c)=\nu(a) \nu(b)$.)

We write $a^{\nu}$ for $\nu(a)$. We write $a \cong_{\nu} b$ for $a \in R_{k}$ and $b \in R_{\ell}$ whenever $a^{\nu}=b^{\nu}$, which means $\nu_{m, k}(a)=\nu_{m, \ell}(b)$ in $R_{m}$ for some $m \geq k, \ell$. (This notation is used generically: We write $a \cong_{\nu} b$ even when the sort transmission maps are denoted differently.) 
Similarly, we write $a \leq_{\nu} b$ if $a^{\nu}+b^{\nu}=b^{\nu}$, which means $\nu_{m, k}(a)+\nu_{m, \ell}(b)=\nu_{m, \ell}(b)$ in $R_{m}$ for some $m \geq k, \ell$, and we write $a<_{\nu} b$ if $a \leq_{\nu} b$ but $a \neq_{\nu} b$.

The axioms are as follows:

A1. $\mathbb{1}_{R} \in R_{1}$.

A2. If $a \in R_{k}$ and $b \in R_{\ell}$, then $a b \in R_{k \ell} \cup R_{0}$.

A3. The product in $R$ is compatible with sort transition maps: Suppose $a \in R_{k}, b \in R_{\ell}$, with $m \geq k$ and $m^{\prime} \geq \ell$. Then

$$
\nu_{m, k}(a) \cdot \nu_{m^{\prime}, \ell}(b)=\nu_{m m^{\prime}, k \ell}(a b) .
$$

A4. $\nu_{\ell, k}(a)+\nu_{\ell^{\prime}, k}(a)=\nu_{\ell+\ell^{\prime}, k}(a)$ for all $a \in R_{k}$ and all $\ell, \ell^{\prime} \geq k$.

A5. If $a \in R_{k}, b \in R_{\ell}$, and $c=a+b \in R_{k^{\prime}}$, then

$$
\nu_{m, k^{\prime}}(c)=\nu_{m, k}(a)+\nu_{m, \ell}(b)
$$

for each $m \geq k+\ell$.

A6. $R_{0}$ is an additive semigroup (and thus an ideal) of $R$.

B. (Supertropicality) Suppose $a \in R_{k}, b \in R_{\ell}$, and $a \cong_{\nu} b$. Then $a+b \in R_{k+\ell}$ with $a+b \cong_{\nu}$ a. If moreover $k=\infty$, then $a+b=a$.

We say that any element a of $R_{k}$ has sort $k(k \in L)$. L is called the sorting semiring of the layered semiring $^{\dagger} R=\bigcup_{\ell \in L} R_{\ell}$. Thus, $\ell$ is the sort of the layer $R_{\ell}$.

The sorting map $s: R \rightarrow L$ is the map that sends every element $a \in R_{\ell}$ to its sort $\ell$.

(Taken from [19, Definition 5.2]) An L-layered pre-domain ${ }^{\dagger}$ is an L-layered semiring ${ }^{\dagger}$ in which Axiom A2 is strengthened to the condition ab $\in R_{k \ell}$. An L-layered semiring $g^{\dagger}:=\left(R, L, s,\left(\nu_{m, \ell}\right)\right)$ is called uniform when the sorting semiring ${ }^{\dagger} L$ is totally ordered and the sort transition maps $\nu_{\ell, k}$ are bijective for each $\ell>k>0$.

Definition 4.2. An L-layered pre-domain ${ }^{\dagger}$ is an L-layered semiring ${ }^{\dagger} R$ for which $R_{1}$ is a monoid.

Definition 4.3. An L-layered semiring ${ }^{\dagger}$ is $\nu$-bipotent if $a+b \in\{a, b\}$ whenever $a \varlimsup_{\nu} b$.

An L-layered bi-domain ${ }^{\dagger}$ is a $\nu$-bipotent L-layered domain ${ }^{\dagger}$.

Remark 4.4. For layered bi-domains ${ }^{\dagger}$, Axiom A5 says that $a<_{\nu} b$ implies $\nu_{m, k}(a)<_{\nu} \nu_{m, \ell}(b)$.

Let us put Construction 3.5 into context, using the layered version of Definition 3.3 An element $z \in R$ is a $\nu$-noncancellative product if $z^{\nu}=a^{\nu} b^{\nu}=a^{\nu} c^{\nu}$ for suitable $a, b, c$, where $b \neq_{\nu} c$. Note that the set of $\nu$-noncancellative products of an $L$-layered semiring ${ }^{\dagger}$ is an ideal. The potential for noncancellative products was one motivation for Construction 3.5 so the next result becomes relevant.

Proposition 4.5. Suppose $z=$ is a $\nu$-noncancellative product, with $\ell=s(z)$. Then $\ell=2 \ell$. In particular, if $\ell$ is finite, then $\ell=0$.

Proof. If ${ }^{[\ell]} z=a b \cong_{\nu} a c$ with $b^{\nu}>c^{\nu}$, then

$$
{ }^{[\ell]} z=a b=a(b+c)=a b+a c={ }^{[\ell]} z+{ }^{[\ell]} z={ }^{[2 \ell]} z,
$$

implying $\ell=2 \ell$.

Since 0 and $\infty$ are multiplicative idempotents of $L$, one could formulate an analogous definition using the layer at $\infty$ instead of at 0 , and indeed this version is implicit in some of our work on superalgebras and supervaluations, such as [20] and 21]. However, there are several good reasons for using the 0 layer in place of the $\infty$ layer.

(1) $R_{\infty}$ corresponds to the image of the ghost map $\nu$, which may involve considerable contraction. On the other hand, we often do not want any contraction to $R_{0}$.

(2) In some ways, $R_{0}$ and $R_{\infty}$ should be complements, as indicated presently.

(3) $R_{0}$ is an ideal which behaves much like a zero element. In particular, it is more intuitive for the zero element (if it exists) to belong to $R_{0}$. 
(4) Remark 4.10 below formalizes the notion that $R_{0}$ also has tangible properties.

Remark 4.6. The 0-layer and the $\infty$-layer behave similarly, since both 0 and $\infty$ are absorbing elements of $L$, except that 0 also absorbs $\infty$ in the sense that $0 \cdot \infty=0$. In case $\infty \in L$ but $0 \notin L, R_{\infty}$ is an ideal of $R$ that can often be used to replace $R_{0}$ in the above discussion.

One difference between the 0 layer and the $\infty$ layer is that for $a \cong{ }_{\nu} b$ with $b \in R_{\ell}$, if $a \in R_{0}$ then $s(a+b)=\ell$, whereas if $a \in R_{\infty}$ then $s(a+b)=\infty$.

Lemma 4.7. The layer $R_{0}$ is also an ideal of $R$. If furthermore $\mathbb{O}_{R} \in R$, then $\mathbb{O}_{R} \in R_{0}$.

Proof. The first assertion is clear. Suppose $\mathbb{O}_{R} \in R_{k}$. Then for any $a \in R_{0}$ we have

$$
\mathbb{O}_{R}=\mathbb{O}_{R} \cdot a \in R_{k \cdot 0}=R_{0} .
$$

Remark 4.8. If $\infty \in L$, then $R_{\infty}$ is a monoid, and $R_{0} \cup R_{\infty}$ is an ideal of $R$.

Lemma 4.9. If $\mathcal{M}$ is any submonoid of a layered semiring ${ }^{\dagger} R:=\left(R, L, s,\left(\nu_{m, \ell}\right)\right)$, then the additive sub-semigroup $\overline{\mathcal{M}}$ of $R$ generated by $\mathcal{M}$ is also a layered semiring ${ }^{\dagger}$.

Proof. $\overline{\mathcal{M}}$ is closed under multiplication, and thus is a semiring ${ }^{\dagger}$. Axiom A1 is given, and the other axioms follow a fortiori.

4.1. The $\{0,1\}$-submonoid. Since in general $R_{1}$ no longer turns out to be a monoid, we must also take into account the 0-layer.

Remark 4.10. $R_{0} \cup R_{1}$ is a submonoid of $R$.

Definition 4.11. The $\{0,1\}$-submonoid is the submonoid of $R$ generated by $R_{1}$.

Thus, the $\{0,1\}$-submonoid is contained in $R_{0} \cup R_{1}$. Since $\mathbb{1}_{R} \in R_{1}$, every invertible element of the fundamental submonoid must lie in $R_{1}$.

Proposition 4.12. Suppose $R$ is an L-layered semiring ${ }^{\dagger}$. Then $\cong_{\nu}$ is an equivalence relation, whose set $\mathcal{G}$ of equivalence classes is a monoid, which is ordered when $R$ is $\nu$-bipotent. In this case, the $\{0,1\}$ submonoid $\mathcal{T}$ of $R$ has an m-valuation $\nu: \mathcal{T} \rightarrow \mathcal{G}$ satisfying $a \mapsto\left[a^{\nu}\right]$.

Proof. $\cong_{\nu}$ is an equivalence relation by definition, and the equivalence classes comprise a monoid in view of Axiom A3. When $R$ is $\nu$-bipotent, we get an ordered monoid by Remark 4.4, and $\nu$ is an m-valuation by Axiom A3.

We are interested in generation by the $\{0,1\}$-submonoid.

Definition 4.13. The tangibly generated sub-semiring ${ }^{\dagger} R_{\langle 1\rangle}$ of an L-layered semiring ${ }^{\dagger} R$ is the subsemiring ${ }^{\dagger}$ generated by $R_{1}$; the semiring ${ }^{\dagger} R$ is tangibly generated if $R_{\langle 1\rangle}=R$.

Thus, $R$ is tangibly generated if $R=\left(\bigcup_{k \in L} \nu_{k, 1}\left(R_{1}\right)\right) \cup R_{0}$.Passing to $R_{\langle 1\rangle}$ may shrink the sorting set.

Lemma 4.14. The tangibly generated sub-semiring ${ }^{\dagger} R_{\langle 1\rangle}$ of a $\nu$-bipotent layered semiring ${ }^{\dagger}$ is a tangibly generated, $\nu$-bipotent layered semiring ${ }^{\dagger}$ with respect to the sorting sub-semiring ${ }^{\dagger}$ of $L$ generated by $1_{L}$. If $R$ is a layered pre-domain ${ }^{\dagger}$, then $R_{\langle 1\rangle}$ is a layered pre-domain ${ }^{\dagger}$ whose 0-layer is empty.

Proof. The axioms are verified a fortiori, since addition only involves adding sorts, starting with $1_{L}$. For the second assertion, since addition cannot lower the sort, we do not get any elements of sort 0 .

Thus, replacing $R$ by its tangibly generated sub-semiring ${ }^{\dagger}$ enables us to assume that $(L,+)$ is generated by 1 and 0 .

Example 4.15. Construction 3.5 is tangibly generated.

It turns out that we could develop the theory under the weaker condition that $L$ is a partially preordered multiplicative monoid, and we sketch the appropriate changes at the end of the Appendix. 
Example 4.16. Given any ideal $\mathfrak{a}$ of an L-layered semiring ${ }^{\dagger} R$, we formally define $R_{\mathfrak{a}}$ to be $R$ with the same semiring $\dagger$ operations, and to have the same sort function as $R$, except that now $s(a)=0$ for every $a \in \mathfrak{a}$. In other words,

$$
\left(R_{\mathfrak{a}}\right)_{0}:=R_{0} \cup \mathfrak{a} ; \quad\left(R_{\mathfrak{a}}\right)_{\ell}:=R_{\ell} \backslash\left(\mathfrak{a} \cap R_{\ell}\right) .
$$

Now define

$$
\overline{\mathfrak{a}}:=\left\{b \in R: b \cong_{\nu} a\right\} .
$$

Then $\overline{\mathfrak{a}} \triangleleft R$, so we could use $\overline{\mathfrak{a}}$ instead of $\mathfrak{a}$.

Proposition 4.17. $R_{\mathfrak{a}}$ is a semiring ${ }^{\dagger}$.

Proof. We need to check associativity and distributivity. But this is clear unless we are using elements of $\mathfrak{a}$, and then associativity holds because all products have layer 0 . Likewise, to see that $a(b+c)$ and $a b+a c$ have the same layer, note this is clear if $s(a)=0$ or if $s(b+c) \neq 0$. Thus we may assume that $s(b+c)=0$, and again we are done if $s(b)=s(c)=0$, so we may assume that $s(b)=0$ and $s(c) \neq 0$ with $b>_{\nu} c$ but $a b \cong_{\nu} a c$. But then

$$
s(a(b+c))=s(a b)+s(a c)=s(a b),
$$

so $a(b+c)=a b=a b+a c$.

Remark 4.18. If $R \backslash \mathfrak{a}$ is finite, then $\left(R_{\mathfrak{a}}\right)_{1}$ is a finite set. Thus, we have a way of "contracting" the tangible component to a finite set.

One instance of arithmetic significance is when $R=\mathscr{R}(L, \mathbb{N} \cup\{0\})$ where $\mathfrak{a}=\left\{{ }^{[\ell]} n: n>q, \ell \in L\right\}$ for some $q \in \mathbb{N}$. In this case, we can "compress" a to a single element in $R_{0}$.

Example 4.19 (The layered $\nu$-truncated semiring ${ }^{\dagger}$ ). Take an ordered semiring $L$ and ordered triple $(\mathcal{M}, \mathcal{G}, v)$, with $R=\mathscr{R}(L \backslash\{0\}, \mathcal{M})$, and fixing $q>\mathbb{1}_{\mathcal{M}}$ in $\mathcal{M}$, define

$$
\mathfrak{a}:=\left\{{ }^{[\ell]} a: v(a) \geq v(q)\right\} \triangleleft R .
$$

Then $R_{\mathfrak{a}}$ contracts to the L-layered semiring ${ }^{\dagger}$

$$
\left\{{ }^{[k]} a: \ell \in L, a<q\right\} \cup\left\{{ }^{[0]} q\right\},
$$

where addition is defined as in Construction [3.5, and multiplication ${ }^{[k]} a{ }^{[\ell]} b$ is given as in Equation (3.3) except for $a b \geq q$, in which case ${ }^{[k]} a{ }^{[\ell]} b={ }^{[0]} q$ for any $k, \ell \in L$. Addition is given by

$$
{ }^{[k]} a+{ }^{[0]} q={ }^{[0]} q .
$$

The sort transition maps are as in Construction [3.5. Thus, ${ }^{[0]} q$ is the special infinite element.

When instead the layering semiring ${ }^{\dagger} L$ is finite, we see that $R_{1} \cup\left\{{ }^{[0]} q\right\}$ is a finite set, which merits further study using arithmetic techniques.

Here is a way to make $L$ finite.

Example 4.20 (The $L$-truncated semiring $\left.{ }^{\dagger}\right)$. Take an ordered semiring $L$ and ordered triple $(\mathcal{M}, \mathcal{G}, v)$, with $R=\mathscr{R}(L \backslash\{0\}, \mathcal{M})$, and fix $m>\mathbb{1}_{\mathcal{M}}$ in $L$. Then $R_{\mathfrak{a}}$ contracts to the L-layered semiring ${ }^{\dagger}$

$$
\left\{{ }^{[\ell]} a: \ell \leq m \in L, a \in \mathcal{M}\right\},
$$

where addition is defined as in Construction [3.5, and multiplication ${ }^{[k]} a{ }^{[\ell]} b$ is given as in Equation (3.3) except for $k \ell \geq m$, in which case ${ }^{[k]} a{ }^{[\ell]} b={ }^{[m]} a b$. Addition is given by

$$
{ }^{[k]} a+{ }^{[m]} \mathbb{\emptyset}={ }^{[k]} a .
$$

The sort transition maps are as in Construction 3.5. Thus, $R_{m}$ is the special infinite layer.

Thus, the two kinds of truncation can interweave to create finite layered structures. 
4.2. The case of onto sort transition maps. We write $e_{\ell}$ for $\nu_{\ell, 1}\left(\mathbb{1}_{R}\right)$. Here is a key simplification for layered domains ${ }^{\dagger}$ when the sort transition maps are onto, which enables us to reduce many results to the tangible case:

Lemma 4.21. If $R$ is an L-layered semiring ${ }^{\dagger}$ and $a \in R_{\ell}$ with $\nu_{\ell, 1}: R_{1} \rightarrow R_{\ell}$ onto, then $a=e_{\ell} a_{1}$ for some $a_{1} \in R_{1}$.

Proof. Taking $a_{1} \in R_{1}$ for which $\nu_{\ell, 1}\left(a_{1}\right)=a$, we have $a=\nu_{\ell, 1}\left(a_{1}\right)=e_{\ell} a_{1}$.

Note 4.22. Lemma 4.21 enables us to simplify the theory for any layer $\ell>1$ for which $\nu_{\ell, 1}$ is onto. When $\ell<1$ we could go in the opposite direction, and define $e_{\ell}$ such that $\nu_{1, \ell}\left(e_{\ell}\right)=\mathbb{1}_{R}$. This will be well-defined when $\nu_{1, \ell}$ is $1: 1$ since, writing $\ell=\frac{m}{n}$ for any $a \in R_{\ell}$ with $\nu_{1, \ell}(a)=\mathbb{1}_{R}$, we have

$$
n e_{\ell}=n e_{m / n}=e_{m}=\nu_{m, \ell}(a)=n a,
$$

implying $a=e_{\ell}$.

4.3. Adjoining the 0-layer. Starting with an $L$-layered pre-domain ${ }^{\dagger} R$ with respect to a semiring ${ }^{\dagger} L$, we can adjoin a zero layer $R_{0}$ formally in several ways. The first way is simply by adjoining a zero element to $R$.

Remark 4.23. For any layered pre-domain ${ }^{\dagger} R$ with respect to a semiring ${ }^{\dagger} L$, the semiring

$$
R \dot{\cup}\left\{\mathbb{O}_{R}\right\}
$$

can be layered with respect to the semiring

$$
L^{0}:=L \dot{\cup}\left\{0_{L}\right\},
$$

where we take $R_{0}:=\left\{\mathbb{O}_{R}\right\}$, putting it in the zero layer as seen by applying the argument of Proposition 4.17. We take the sort transition maps $\nu_{0, \ell}(a):=\mathbb{O}_{R}$ for all $\ell \neq 0$ and $a \in R$.

However, this is not the only possibility for the zero layer, as we saw in [17, Remark 3.8].

Construction 4.24. If $R$ is a uniform L-layered pre-domain ${ }^{\dagger}$, where $L$ is a semiring ${ }^{\dagger}$, then adjoining $\{0\}$ formally to $L$ as the unique minimal element, we can form a uniform $L^{0}$-layered semiring ${ }^{\dagger} R \cup R_{0}$, where $R_{0}:=e_{0} R_{1}$ is another copy of $R_{1}$, under the same rules of addition and multiplication given by Construction 3.2.

Proof. If $a=e_{0} a_{1}, b=e_{k} b_{1}$, and $c=e_{\ell} c_{1}$ for $a_{1}, b_{1}, c_{1} \in R_{1}$, then

$$
(a b) c=e_{0} e_{k} e_{\ell}\left(a_{1} b_{1}\right) c_{1}=e_{0} e_{k} e_{\ell} a_{1}\left(b_{1} c_{1}\right)=e_{0} a_{1}\left(b_{1} c_{1}\right)=e_{0} a_{1}\left(b_{1} c_{1}\right)=a(b c),
$$

yielding associativity of multiplication. To see distributivity, we note that $e_{k} b_{1}+e_{\ell} c_{1}=e_{m}\left(b_{1}+c_{1}\right)$ where $m \in\{k, \ell, k+\ell\}$, so

$$
a(b+c)=e_{0} e_{m} a_{1}\left(b_{1}+c_{1}\right)=e_{0} a_{1}\left(b_{1}+c_{1}\right)=e_{0} a_{1} b_{1}+e_{0} a_{1} c_{1}=e_{0} e_{k} a_{1} b_{1}+e_{0} e_{\ell} a_{1} c_{1}=a b+a c .
$$

Associativity of addition is similar. Finally, if $a=\mathbb{O}_{R} \in R_{0}$ and $b \in R_{\ell}$, then $a b \in R_{0 \cdot \ell}=R_{0}$.

Since we have several ways of adjoining a zero layer, the following observation is useful.

Proposition 4.25. For any semiring $R$ layered with respect to a semiring ${ }^{\dagger} L, R \dot{\cup}\left\{\mathbb{O}_{R}\right\}$ is an $L^{0}$-layered sub-semiring of $R \dot{\cup} R_{0}$.

More generally, for any ideal $\mathfrak{a}$ of $R$, writing $\mathfrak{a}_{0}$ for $\mathfrak{a} \cap R_{0}$, we have $\left(\bigcup_{\ell \neq 0} R_{\ell}\right) \cup \mathfrak{a}_{0}$ is an $L^{0}$-layered sub-semiring of $R \dot{\cup} R_{0}$.

Proof. If $a \in \mathfrak{a}_{0}$ and $b \in R_{\ell}$, then $a b \in R_{0 \cdot \ell}=R_{0}$, implying $a b \in \mathfrak{a}_{0}$.

This gives rise to the question of whether we should adjoin the entire 0-layer, or just $\mathbb{O}_{R}$ ? Although one's experience from classical algebra might lead one to adjoin only $\mathbb{O}_{R}$, there are situations in which one might need other elements in $R_{0}$ in order to distinguish polynomials. 
4.4. Adjoining the absolute ghost layer, and the passage to standard supertropical domains ${ }^{\dagger}$. Even when $L$ originally does not contain an infinite element a priori, $L$-layered bi-domains ${ }^{\dagger}$ tie in directly with the (standard) supertropical theory, via a ghost layer introduced at a new element $\infty$ which we adjoin. (This works even when $(\geq)$ is merely a partial order on $L$, although it it is easier when $(\geq)$ is a total order.)

Remark 4.26. Any L-layered semiring ${ }^{\dagger}\left(R, L, s,\left(\nu_{m, \ell}\right)\right)$ is a directed system with respect to the set $L$, as described in [23, p. 71]. Hence, by [23, Theorem 2.8], the layers $R_{k}$ have a direct limit which we denote $R_{\infty}$, and maps

$$
\nu_{\infty, k}: R_{k} \rightarrow R_{\infty}
$$

such that $\nu_{\infty, k}=\nu_{\infty, \ell} \circ \nu_{\ell, k}$ for each $a \in R_{k}$ and all $k<\ell$. Since $R=\bigcup_{k} R_{k}$, we can piece together these maps $\nu_{\infty, k}$ to a map $\nu: R \rightarrow R_{\infty}$. We define

$$
e=e_{\infty}:=\nu\left(\mathbb{1}_{R}\right)
$$

easily seen to be the unit element of $R_{\infty}$.

We write $a^{\nu}$ for $\nu(a) \in R_{\infty}$. Thus $a^{\nu}=b^{\nu}$ iff $a \cong_{\nu} b$ in our previous notation.

We call $R_{\infty}$ the absolute ghost layer and $\nu$ the (absolute) ghost map of $R$. Note that in the uniform case, $R_{\infty}$ is just another copy of $R_{1}$, so we can dispense with direct limits.

Theorem 4.27. Suppose $R:=\left(R, L, s,\left(\nu_{m, \ell}\right)\right)$ is an L-layered semiring ${ }^{\dagger}$. Then the absolute ghost layer $R_{\infty}$ is a bipotent semiring ${ }^{\dagger}$. The ghost map $\nu: R \rightarrow R_{\infty}$ is a semiring $g^{\dagger}$ homomorphism. Define

$$
U=U(R):=R \dot{U} R_{\infty} .
$$

Then $U$ is a semiring ${ }^{\dagger}$ under the given operations of $R$ and $R_{\infty}$, together with

$$
\begin{aligned}
a \cdot b^{\nu} & :=(a b)^{\nu} ; \\
a+b^{\nu} & := \begin{cases}a & \text { if } e a>e b, \\
b^{\nu} & \text { if } e a \leq e b .\end{cases}
\end{aligned}
$$

Also, extend $\nu$ to a map $\nu_{U}: U \rightarrow R_{\infty}$ by taking $\nu_{U}$ to be the identity on $R_{\infty}$. Then $U$ has ghost ideal $\mathcal{G}=\mathcal{G}(U):=R_{\infty}$, in the sense of [21], and $\nu_{U}(a)=$ ea for every $a$ in $U$.

Then $U$ can be modified to a supertropical semiring ${ }^{\dagger}$

$$
\mathcal{R}_{1, \infty}:=R_{1} \dot{\cup} \mathcal{G},
$$

retaining the given multiplication - of $U$, but with new addition $\oplus$ given by the rules

$$
a \oplus b:= \begin{cases}a & \text { if } e a>e b, \\ b & \text { if } e a<e b, \\ e a & \text { if } e a=e b .\end{cases}
$$

Proof. Axiom A3 tells us that

$$
\nu_{m m^{\prime}, k \ell}(a \cdot b)=\nu_{m, k}(a) \cdot \nu_{m^{\prime}, \ell}(b)
$$

for any $a \in R_{k}$ and $b \in R_{\ell}$; taking limits yields

$$
\nu(a \cdot b)=\nu(a) \cdot \nu(b) .
$$

Likewise, Axiom B tells us that

$$
\nu(a+b)=\nu(a)+\nu(b) .
$$

The other verifications are also easy. By (4.3) we have

$$
\nu(x)=e \cdot x \quad \text { for every } \quad x \in R .
$$

Thus $\nu \circ \nu=\nu$, and also $\nu: R \rightarrow \mathcal{G}$ is a semiring ${ }^{\dagger}$ homomorphism from $R$ onto $\mathcal{G}=\mathcal{G}(U)$.

We extend the $\nu$-equivalence relation from $R$ to $U$ by decreeing that $a \equiv_{U} b$ iff $a$ and $b$ have the same value under $\nu$.

We turn to the last assertion. Due to (4.4) we have

$$
a \oplus b=a+b \quad \text { if } \quad a \neq_{\nu} b .
$$


On the other hand,

$$
a \oplus b=e(a+b) \text { if } \quad a \cong_{\nu} b
$$

Note that

$$
a \oplus b \cong_{\nu} a+b
$$

in all cases. Also, $\mathcal{G}(U):=R_{\infty}=\mathcal{G}\left(\mathcal{R}_{1, \infty}\right)$.

We may regard $\mathcal{R}_{1, \infty}:=\left(\mathcal{R}_{1, \infty}, \oplus, \cdot\right)$ as a degeneration of the semiring ${ }^{\dagger} U:=U(R)$, where all the ghost layers have been coalesced to $R_{\infty}$. When $L=L_{\geq 1}$, then there is a semiring ${ }^{\dagger}$ homomorphism $U \rightarrow \mathcal{R}_{1, \infty}$ given by

$$
a \mapsto \begin{cases}a & \text { for } \quad a \in R_{1} \cup R_{\infty}, \\ \nu(a) & \text { otherwise. }\end{cases}
$$

We are now in a position to see why Construction 3.2 of a uniform $L$-pre-domain ${ }^{\dagger}$ is generic. We recall

$$
R(L, \mathcal{G}):=\left\{{ }^{[\ell]} a \mid a \in \mathcal{G}, \ell \in L\right\} .
$$

Remark 4.28. In a uniform L-layered pre-domain ${ }^{\dagger}$, we can define $\nu_{k, \ell}$ for $0<k<\ell$ to be $\nu_{\ell, k}^{-1}$. Thus, $\nu_{k, \ell}$ is defined for all $0<k, \ell \in L$.

\section{MORPhisms OF LAYERED SEMIRINGS ${ }^{\dagger}$}

In ordered to understand layered categories, we need a good notion of morphism. This is easiest to describe for layered domains ${ }^{\dagger}$.

5.1. Layered homomorphisms. We assume that $L$ is non-negative.

Definition 5.1. A layered homomorphism

$$
(\varphi, \rho):\left(R, L, s,\left(\nu_{m, \ell}\right)\right) \rightarrow\left(R^{\prime}, L^{\prime}, s^{\prime},\left(\nu_{m, \ell}^{\prime}\right)\right)
$$

of uniform $L$-layered pre-domains ${ }^{\dagger}$ is a semiring ${ }^{\dagger}$ homomorphism $\rho: L \rightarrow L^{\prime}$ preserving the given partial orders, i.e., satisfying the condition:

M1. $k \leq \ell$ implies $\rho(k) \leq \rho(\ell)$.

together with a semiring homomorphism $\varphi: R \rightarrow R^{\prime}$ such that

M2. $\mathrm{s}^{\prime}(\varphi(a)) \geq \rho(\mathrm{s}(a)), \quad \forall a \in R$.

The definition becomes more complicated when $0 \in L$; then we need to modify Axiom M2 to:

$$
\text { M2'. } \mathrm{s}^{\prime}(\varphi(a))=\ell \text {, where } \ell=0 \text { or } \ell \geq \rho(\mathrm{s}(a)), \quad \forall a \in R \text {. }
$$

We always write $\Phi:=(\varphi, \rho)$. We often assume $L=L^{\prime}$ and $\rho=\mathrm{id}_{L}$; we call $\Phi$ a natural homomorphism in this situation.

Lemma 5.2. Write $e_{\ell, R}$ for $e_{\ell}$ in $R$. Then $\varphi\left(e_{\ell, R}\right)=e_{\ell, R^{\prime}}$, for each $\ell$ in the sub-semiring ${ }^{\dagger}$ of $L$ (resp. $\left.L^{\prime}\right)$ generated by 1 .

Proof. Then $\varphi\left(e_{1, R}\right)=\varphi\left(\mathbb{1}_{R}\right)=\mathbb{1}_{R^{\prime}}=e_{1, R^{\prime}}$. Thus, for each $n \in \mathbb{N}$, we have

$$
\varphi\left(e_{n, R}\right)=\varphi\left(e_{1, R}+\cdots+e_{1, R}\right)=\varphi\left(e_{1, R}\right)+\cdots+\varphi\left(e_{1, R}\right)=e_{1, R^{\prime}}+\cdots+e_{1, R^{\prime}}=e_{n, R^{\prime}} .
$$

It follows at once that the homomorphism $\varphi$ is given by its action on $R_{1}$.

Proposition 5.3. If $a=e_{\ell} a_{1}$ as in Lemma 4.21, then

$$
\varphi(a)=\varphi\left(e_{\ell, R}\right) \varphi\left(a_{1}\right)=e_{\ell, R^{\prime}} \varphi\left(a_{1}\right), \quad \forall \ell>0 .
$$

Proof. $\varphi(a)=\varphi\left(e_{\ell, R}\right) \varphi\left(a_{1}\right)=e_{\ell, R^{\prime}} \varphi\left(a_{1}\right)$.

Corollary 5.4. Equation (5.1) holds automatically whenever $R$ is uniform L-layered. 
Proof. Lemma 4.21 is applicable.

Proposition 5.5. Suppose $\varphi: R \rightarrow R^{\prime}$ is a layered homomorphism, and $R$ is tangibly generated. Then $\varphi$ is determined by its action on $R_{0} \cup R_{1}$, via the formula

$$
\varphi\left(\sum_{i} a_{i}\right)=\sum_{i} \varphi\left(a_{i}\right) .
$$

Proof. It is enough to check sums, in view of Lemma 4.9.

Our category will be comprised of the tangibly generated $L$-layered semirings ${ }^{\dagger}$.

5.2. Layered supervaluations and the layered analytification. In case our layered semiring ${ }^{\dagger}$ is not uniform, we need a more general notion of morphism, treated in [19. To understand what is going on, we need to generalize the notion of "valuation." Valuations are important in algebraic geometry, and play a key role in tropical theory largely because of the following example.

Example 5.6. Recall that the field $\mathbb{K}$ of Puiseux series over an algebraically closed field $F$ is defined to be the set of all series of the form

$$
p(t):=\sum_{\tau \in T} c_{\tau} t^{\tau}, \quad c_{\tau} \in F,
$$

with $T \subset \mathbb{Q}$ well-ordered and bounded from below, endowed with the valuation Val : $\mathbb{K}^{\times} \longrightarrow \mathbb{R}$ given by

$$
\operatorname{Val}(p(t)):=-\min \left\{\tau \in T: c_{\tau} \neq \mathbb{O}_{F}\right\}, \quad p(t) \in \mathbb{K} \backslash\left\{\mathbb{O}_{\mathbb{K}}\right\} .
$$

A word about notation: Given a valuation (or, more generally, an m-valuation) $v$, one can replace $v$ by $-v$ and reverse the customary inequality to get

$$
v(a+b) \leq \max \{v(a), v(b)\},
$$

which is more compatible with the max-plus set-up.

Payne 28] has developed an algebraic version of Berkovich's theory of analytification, which can be viewed as the limit of tropicalizations. In his theory, a multiplicative seminorm ||$: W \rightarrow \mathbb{R}$ on a ring $W$ is a multiplicative map satisfying the triangle inequality

$$
|a+b| \leq|a|+|b| \text {. }
$$

The underlying space in Payne [27] is the set of multiplicative seminorms from $K\left[\lambda_{1}, \ldots, \lambda_{n}\right]$ to $\mathbb{R}_{>0}$ extending $v$, for a given m-valuation $v: K \rightarrow \mathbb{R}_{>0}$. We generalize this definition by taking an arbitrary ordered semiring ${ }^{\dagger}$ instead of $\mathbb{R}_{>0}$.

The supertropical version, the strong supervaluation, is defined in [16, Definition 4.1 and Definition 9.9] as a monoid homomorphism $\varphi$ satisfying $\varphi(a)+\varphi(b) \underset{\text { gs }}{\models} \varphi(a+b)$, where $\underset{\text { gs }}{\models}$ is the ghost surpassing relation of [16, Definition 9.1]. In this way, strong supervaluations generalize seminorms.

Here is the layered analog.

Definition 5.7. A layered supervaluation on a ring $W$ is a map $\varphi: W \rightarrow R$ from $W$ to an $L$-layered semiring $R$ with the following properties:

$$
\begin{aligned}
& L V 1: \varphi(1)=\mathbb{1}_{R}, \\
& L V 2: \forall a, b \in R: \varphi(a b)=\varphi(a) \varphi(b), \\
& L V 3: \forall a, b \in R: \varphi(a+b) \leq_{\nu} \varphi(a)+\varphi(b), \\
& L V 4: \varphi(0)=\mathbb{O}_{R} .
\end{aligned}
$$

$A\{0,1\}$-layered supervaluation on a ring $W$ is a layered supervaluation $\Phi: W^{\times} \rightarrow R$, where $W^{\times}:=W \backslash\{0\}$, such that $\Phi(W) \subseteq R_{0} \cup R_{1}$.

Proposition 5.8. Suppose that $R:=\mathscr{R}(L, \mathcal{G})$ an L-layered bi-domain ${ }^{\dagger}$. If $\Phi: W \rightarrow \mathcal{G}$ is a $\{0,1\}$-layered supervaluation of a ring $W$, then $\Phi(a)$ is tangible for every invertible element $w$ of $W$. (In particular, if $W$ is a field, then $\Phi\left(W^{\times}\right)$is tangible. $)$

Proof. $\Phi(w) \Phi\left(w^{-1}\right)=\Phi(1)=\mathbb{1}_{R}$, so $\Phi(w) \notin R_{0}$, and thus is tangible. 
In this situation, the tangible layer determines the layered supervaluation.

The morphisms in the layered category should then be those maps which transfer one layered supervaluation to another. In the standard supertropical situation, these are the transmissions of [18, which are given in the layered setting in [19]. This paves the way for the following concept, with, notation as in Example 5.6

Remark 5.9. Let $R:=\mathscr{R}(L, \mathcal{G})$, and view Val as the composite map of monoids

$$
\mathbb{K} \stackrel{\text { Val }}{\rightarrow} \mathcal{G} \cong R_{1} \subseteq R
$$

Then for any affine algebraic variety $X$ over $\mathbb{K}$, the space of $\{0,1\}$-layered valuations from $\mathbb{K}\left[\lambda_{1}, \ldots, \lambda_{n}\right]$ to $R$ extends $\mathbb{K}^{\text {an }}$ of $[28$, and its theory invites further study.

5.3. Surpassing and surpassed maps. In line with the philosophy of this paper, we would like to introduce the category of $L$-layered semirings ${ }^{\dagger}$. Having the layered semirings ${ }^{\dagger}$ in hand, we next need to define the relevant morphisms. From now on, to avoid complications, we assume that $R$ is a uniform, $L$ layered pre-domain ${ }^{\dagger}$. As indicated in the introduction, although the natural definition from the context of semirings ${ }^{\dagger}$ is good enough for most purposes, a sophisticated analysis requires us to consider the notion of "supervaluation," and how this would relate to morphisms that preserve the properties of supervaluations, which we will discuss in 97 , But a more naive approach suits our needs in many situations.

5.3.1. The surpassing relation. For $\ell \in L$, an $\ell$-ghost sort is an element of the form $\ell+k$, for positive $k \in L$. We say $a$ is $\ell$-ghost if $s(a)$ is an $\ell$-ghost sort. Note that the infinite element $\infty$ of $L$ is a "self-ghost sort," in the sense that $\infty+m=\infty$ implies that $\infty$ is an $\infty$-ghost sort.

Here is a key relation in the theory.

Definition 5.10. The surpassing L-relation $\underset{L}{\models}$ is given by

$$
a \underset{L}{a=b} \quad \text { iff either }\left\{\begin{array}{l}
a=b+c \quad \text { with } \quad c \quad s(b) \text {-ghost }, \\
a=b, \\
a \cong{ }_{\nu} b
\end{array} \quad \text { with } a r\right. \text { s(b)-ghost. }
$$

It follows that if $a \underset{L}{\models} b$, then $a+b$ is $s(b)$-ghost. When $a \neq b$, this means $a \geq \nu b$ and $a$ is $s(b)$-ghost.

Definition 5.11. The surpassing $(L, \nu)$-relation $\underset{L}{\models_{\nu}}$ is given by

$$
a \models_{L}{ }_{\nu} b \quad \text { iff } \quad a \underset{L}{\models} b \text { and } a \cong{ }_{\nu} b .
$$

Lemma 5.12. The surpassing L-relation $\underset{L}{\models}$ and the surpassing $(L, \nu)$-relation $\underset{L}{\models}{ }_{\nu}$ are half-congruences. Proof. Pointwise verifications.

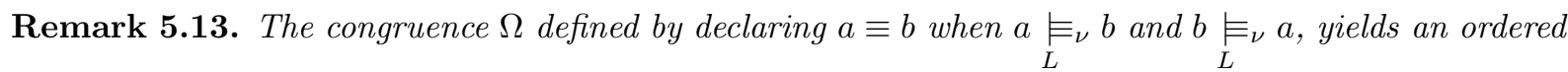
monoid.

5.3.2. Surpassing morphisms. We also weaken the notion of layered homomorphism for layered semirings ${ }^{\dagger}$.

Definition 5.14. A surpassing $\operatorname{map} \varphi: R \rightarrow R^{\prime}$ is a (multiplicative) monoid homomorphism such that $\varphi(a+b) \underset{L}{\equiv} \equiv_{\nu} \varphi(a)+\varphi(b)$.

A surpassed map $\varphi: R \rightarrow R^{\prime}$ is a monoid homomorphism such that $\varphi(a)+\varphi(b) \equiv \equiv_{\nu} \varphi(a+b)$.

A o-excepted homomorphism $\varphi: R \rightarrow R^{\prime}$ is a monoid homomorphism such that $\varphi(a)+\varphi(b)=$ $\varphi(a+b)$ whenever $s(a), s(b)>0$.

(In other words, a 0-excepted homomorphism could fail to be a semiring ${ }^{\dagger}$ homomorphism only because of the behavior of the 0 sort.)

We write $R_{\geq \ell}$ for $\cup_{k \geq \ell} R_{k}$. 
Example 5.15. If $a>_{\nu} b$, then $(a+b)^{m}=a^{m}$. Hence, for any given $m$, the Frobenius property

$$
(a+b)^{m} \underset{L}{\models}{ }_{\nu} a^{m}+b^{m}
$$

from [17, Remark 5.26] is satisfied in any L-layered semiring ${ }^{\dagger}$ and, the Frobenius map $a \mapsto a^{m}$ is a surpassing map in $R_{\geq 1} \cup R_{0}$.

Proposition 5.16. We have the surpassing map $\varphi: M_{n}(R) \rightarrow M_{n}(R)$ given by $\left(a_{i, j}\right) \mapsto\left(a_{i, j}{ }^{m}\right)$.

Proof. We need to show that $\left(c_{i, j}^{m}\right)=\left(a_{i, j}^{m}\right)\left(b_{i, j}^{m}\right)$, where $c_{i, j}=\sum_{k} a_{i, k} b_{k, j}$. But by (5.6),

$$
c_{i, j}{ }^{m}=\left(\sum_{k} a_{i, k} b_{k, j}\right)^{m} \underset{L}{\models} \sum_{k}\left(a_{i, k} b_{k, j}\right)^{m}=\sum_{k} a_{i, k}{ }^{m} b_{k, j}{ }^{m} .
$$

Example 5.17. In the standard supertropical situation, the supertropical determinant (i.e., the permanent) is a surpassing map, by [22].

Proposition 5.18. Any surpassing map $\varphi$ preserves $\nu$, in the sense that if $a \geq_{\nu} b$, then $\varphi(a) \geq_{\nu} \varphi(b)$.

Proof. $\varphi(a) \cong_{\nu} \varphi(a+b) \underset{L}{\equiv_{\nu}} \varphi(a)+\varphi(b)$, implying $\varphi(a) \geq_{\nu} \varphi(b)$.

Nevertheless, we take the morphisms in this category to be the 0-excepted homomorphisms.

5.4. Layered morphisms. Since morphisms lie at the heart of category theory, the time has come to consider the morphisms that arise for layered semirings ${ }^{\dagger}$.

Definition 5.19. A layered morphism of L-layered semirings ${ }^{\dagger}$ is a map

$$
\Phi:=(\varphi, \rho):\left(R, L, s,\left(\nu_{m, \ell}\right)\right) \rightarrow\left(R^{\prime}, L^{\prime}, s^{\prime},\left(\nu_{m^{\prime}, \ell^{\prime}}^{\prime}\right)\right)
$$

where $\rho: L \rightarrow L^{\prime}$ is a semiring $\dagger^{\dagger}$ homomorphism, together with a 0-excepted homomorphism $\varphi: R \rightarrow R^{\prime}$ such that

$$
\begin{aligned}
& \text { M1. } \mathrm{s}^{\prime}(\varphi(a)) \geq \rho(\mathrm{s}(a)) \text { or } \mathrm{s}^{\prime}(\varphi(a))=0 \text {. } \\
& \text { M2. For all } a \in R_{k}, \mathrm{~s}\left(\varphi\left(\nu_{\ell, k}\right)(a)\right) \cong_{\nu} \mathrm{s}(\varphi(a)) \text { for all } \ell \geq k \text {. } \\
& \text { M3. If } \left.a \cong_{\nu} b \text {, then } \varphi(a) \cong_{\nu} \varphi(b) \text { (taken in the context of the } \nu_{m^{\prime}, \ell^{\prime}}^{\prime}\right) \text {. }
\end{aligned}
$$

A layered homomorphism is a layered morphism such that $\varphi: R \rightarrow R^{\prime}$ is a semiring ${ }^{\dagger}$ homomorphism.

We always write $\Phi:=(\varphi, \rho):\left(R, L, s,\left(\nu_{m, \ell}\right)\right) \rightarrow\left(R^{\prime}, L^{\prime}, s^{\prime},\left(\nu_{m^{\prime}, \ell^{\prime}}^{\prime}\right)\right)$, denoted as $\Phi: R \rightarrow R^{\prime}$ when unambiguous. In most of the following examples, the sorting semirings ${ }^{\dagger} L$ and $L^{\prime}$ are the same.

Example 5.20. Here are some examples of layered homomorphisms. We assume throughout that $R$ is an L-layered semiring ${ }^{\dagger}$, although sometimes we consider the role of $\mathbb{O}_{R}$ if it exists.

(a) In the max-plus situation, when $L=\{1\}, \rho$ must be the identity, and $\Phi$ is just a semiring ${ }^{\dagger}$ homomorphism. When $L=\{0,1\}$ and $R_{0}=\left\{\mathbb{O}_{R}\right\}$, we must have $\varphi\left(\mathbb{O}_{R}\right)=\mathbb{O}_{R}$.

(b) In the "standard supertropical situation without 0 ," when $L=\{1, \infty\}, \Phi\left(R_{\infty}\right)=R_{\infty}$.

(c) In the "standard supertropical situation with 0 ," when $L=\{0,1, \infty\}$, and $R_{0}=\left\{\mathbb{O}_{R}\right\}, \Phi$ must send the ghost layer $R_{\infty}$ to $R_{\infty} \cup R_{0}$. If $\mathfrak{a} \triangleleft R$ and $\mathfrak{a} \supset R_{\infty}$, one could take $R^{\prime}=R$ as a set, with $R_{1}^{\prime}=R_{1} \backslash \mathfrak{a}$ and $R_{0}^{\prime}=\mathfrak{a}$. The identity map is clearly a layered homomorphism; its application "expands the zero level" to $\mathfrak{a}$.

(d) Notation as in Theorem [3.6, we define a layered homomorphism $\mathscr{R}(L, \mathcal{G}) \rightarrow \mathscr{R}(L, \mathcal{G})_{\mathfrak{a}}$ given by the identity map on all elements of $\mathscr{R}(L, \mathcal{G}) \backslash \mathfrak{a}$, and ${ }^{[\ell]} a \mapsto{ }^{[0]} a$ for every $a \in \mathfrak{a}$.

(e) Any semiring homomorphism $\rho: L \rightarrow L^{\prime}$ induces a layered homomorphism $\mathscr{R}(L, \mathcal{G}) \rightarrow \mathscr{R}\left(L^{\prime}, \mathcal{G}\right)$ given by ${ }^{[\ell]} a \mapsto{ }^{[\rho(\ell)]} a$.

(f) The natural injections $R_{\geq 1} \cup R_{0} \rightarrow R$ and $\left\{\bigcup_{\ell} R_{\ell}: \ell \in \mathbb{N}\right\} \rightarrow R$ are both examples of layered homomorphisms.

(g) The truncation maps of Example 4.19 and Example 4.20 are layered homomorphisms. 
(h) Suppose $\mathfrak{a} \triangleleft R$ is a $\nu$ - "upper" ideal in $R_{>1}$ or in $R_{>1} \cup R_{0}$, by which we mean an ideal of the form $\left\{r: r \geq_{\nu} a\right\}$ or $\left\{r: r>_{\nu} a\right\}$. We define the congruence $\Omega_{\mathfrak{a}}$ on $R_{\mathfrak{a}}$ to be $(\mathfrak{a} \times \mathfrak{a}) \cup \operatorname{diag}(R)$; in other words, $b_{1} \equiv_{\mathfrak{a}} b_{2}$ if $b_{1} \cong_{\nu} b_{2}$ or if $b_{1}, b_{2} \in \mathfrak{a}$. Then $R_{\mathfrak{a}} / \Omega_{\mathfrak{a}}$ is a layered semiring ${ }^{\dagger}$, under the induced multiplication and addition of equivalence classes, and $a \mapsto[a]$ defines a layered homomorphism. Note that all elements of $\mathfrak{a}$ collapse to a single element, as in the Rees quotient construction for semigroups.

Having these examples in hand, one might wonder why we bother with 0 -excepted homomorphisms in the definition of morphism. This is in order to make Theorem 6.3 possible.

Proposition 5.21. Any layered morphism $\varphi$ on a tangibly generated layered semiring ${ }^{\dagger}$ is determined by its action on the tangible submonoid $R_{0} \cup R_{1}$.

Proof. Since $\varphi\left(e_{k} a\right)=\varphi\left(e_{k}\right) \varphi(a)$, it suffices to check that $\varphi\left(e_{k}\right)$ is uniquely defined. Write

$$
e_{k}^{\prime}=\varphi\left(\mathbb{1}_{R}\right)+\cdots+\varphi\left(\mathbb{1}_{R}\right),
$$

taken $k$ times, whose sort is $k$. Since $\varphi\left(\mathbb{1}_{R}\right)=\mathbb{1}_{R^{\prime}}$, we have $e_{k}^{\prime} \underset{L}{\equiv_{\nu}} \varphi\left(e_{k}\right)$ by definition of 0-excepted homomorphism. Hence, $s\left(\varphi\left(e_{k}\right)\right) \leq k$. But $s\left(\varphi\left(e_{k}\right)\right) \geq k$ by Condition M1, implying $s\left(\varphi\left(e_{k}\right)\right)=k$, and thus $e_{k}^{\prime}=\varphi\left(e_{k}\right)$, as desired.

\section{The LAYERED CATEGORIES AND THEIR TROPICALIZATION FUNCTORS}

Having assembled the basic concepts, we are finally ready to tie these ideas to tropicalization, by introducing the layered categories. Our objective in this section is to introduce the functor that passes from the "classical algebraic world" of integral domains with valuation to the "layered world," taking the cue from [21, Definition 2.1], which we recall and restate more formally.

6.1. Identifications of categories of valued monoids and layered semirings ${ }^{\dagger}$. Here is our main layered category.

Definition 6.1. LaySemi $^{\dagger}$ is the category whose objects are tangibly generated layered semirings ${ }^{\dagger}$ and whose morphisms are layered morphisms.

Remark 6.2. In view of Theorem [3.6] we can define the forgetful functor LaySemi ${ }^{\dagger} \rightarrow \mathrm{OMon}^{+}$given by sending the L-layered semiring ${ }^{\dagger}$ to $R_{0} \cup R_{1}$.

Thus, any layered homomorphism yields a homomorphism of the underlying monoid of tangible elements, thereby indicating an identification between categories arising from the construction of layered pre-domains $^{\dagger}$ from ordered monoids (and more generally, of layered semirings ${ }^{\dagger}$ from valued monoids). But to get the other direction, we need to permit morphisms merely to be surpassed maps, as previously defined.

Theorem 6.3. For any valued semiring $L$, there is a faithful layering functor $\mathcal{F}:$ ValMon $\rightarrow$ LaySemi ${ }^{\dagger}$, given by sending $\mathcal{M}$ to $\mathscr{R}(L, \mathcal{M})_{\overline{\mathfrak{a}}}$, where $\mathfrak{a}$ is the monoid ideal of noncancellative products, and the ordered homomorphism $\varphi: \mathcal{M} \rightarrow \mathcal{M}^{\prime}$ to the layered homomorphism $\mathcal{F} \varphi: \mathscr{R}(L, \mathcal{M}) \rightarrow \mathscr{R}\left(L, \mathcal{M}^{\prime}\right)$ obtained from $\varphi$ as follows:

$\mathcal{F} \varphi$ is defined on $R_{0} \cup R_{1}$ via $\mathcal{F} \varphi\left({ }^{[\ell]} a\right)={ }^{\left[\ell^{\prime}\right]} \varphi(a)$, where $\ell^{\prime}=1$ unless $\varphi(a)$ is a noncancellative product in $\mathcal{G}^{\prime}$, in which case $\ell^{\prime}=0$.

The functor $\mathcal{F}$ is a left retract of the forgetful function of Remark 6.2

Proof. The image of an ordered monoid $\mathcal{G}$ is a layered semiring ${ }^{\dagger}$, in view of Proposition 4.12, and one sees easily that $\mathcal{F} \varphi$ is a layered morphism since, for $a \geq_{\nu} b$,

$$
\mathcal{F} \varphi\left({ }^{[k]} a+{ }^{[\ell]} b\right) \cong_{\nu} \mathcal{F} \varphi\left({ }^{[k]} a\right) \cong_{\nu} \varphi\left({ }^{[k]} a\right) \cong_{\nu} \varphi\left({ }^{[k]} a\right)+\varphi\left({ }^{[\ell]} b\right),
$$

and $s^{\prime}\left(\mathcal{F} \varphi\left({ }^{[k]} a\right)\right)$ is $k$ or 0 .

One needs to verify that $\overline{\mathfrak{a}} R_{1} \subseteq \overline{\mathfrak{a}}$. But $\mathfrak{a} R_{1} \subseteq \mathfrak{a}$ is clear by definition of noncancellative product, yielding $\overline{\mathfrak{a}} R_{1} \subseteq \overline{\mathfrak{a}}$.

The morphisms match. The functor $\mathcal{F}$ is faithful, since one recovers the original objects and morphisms by applying the forgetful functor of Remark 6.2 . 
6.1.1. The layered tropicalization functor.

Definition 6.4. Given a semiring ${ }^{\dagger} L$, the L-tropicalization functor

$$
\mathcal{F}_{\text {LTrop }}: \text { ValMon } \longrightarrow \text { LaySemi }^{\dagger}
$$

from the category of valued monoids to the category of uniform layered semirings ${ }^{\dagger}$ is defined as follows: $\mathcal{F}_{\text {LTrop }}:(\mathcal{M}, \mathcal{G}, v) \mapsto \mathscr{R}(L, \mathcal{G})_{\mathfrak{a}}$ and $\mathcal{F}_{\text {LTrop }}: \phi \mapsto \alpha_{\phi}$, where $\boldsymbol{a}$ is the ideal of noncancellative elements of the monoid $\mathcal{G}$, and, given a morphism $\phi:(\mathcal{M}, \mathcal{G}, v) \rightarrow\left(\mathcal{M}^{\prime}, \mathcal{G}^{\prime}, v^{\prime}\right)$ we define $\alpha_{\phi}: \mathscr{R}(L, \mathcal{G}) \rightarrow \mathscr{R}\left(L^{\prime}, \mathcal{G}^{\prime}\right)$, by

$$
\alpha_{\phi}\left({ }^{[\ell]} a\right):={ }^{[k]} \phi(a), \quad a \in \mathcal{G},
$$

where $k=0$ if $\phi(a)$ is noncancellative and $k=\ell$ if $\phi(a)$ is cancellative, cf. Formula (2.5).

Note that the $L$-tropicalization functor $\mathcal{F}_{\text {LTrop }}$ factors as

$$
\text { ValMon } \rightarrow \text { OMon } \rightarrow \text { LaySemi }
$$

which restricts to ValMon ${ }^{+} \rightarrow$ OMon $^{+} \rightarrow$ LaySemi $^{\dagger}$ of [19].

Suppose $v: W^{\times} \rightarrow \mathcal{G}$ is a valuation on an integral domain $W$, where $W^{\times}:=W \backslash\left\{\mathbb{O}_{W}\right\}$. Let $\mathcal{M}:=W^{\times}$, a multiplicative monoid. Fix $\ell \in L$; usually $\ell=1$. The restriction of $v$ to $\mathcal{M}$, which we denote as $\psi_{\ell}$, can be realized as the map sending $\mathcal{M}$ as a set into the $\ell$-layer of $\mathscr{R}(L, \mathcal{G})$, given by $\psi_{\ell}: a \mapsto{ }^{[k]} v(a)$, where $k=0$ if $a$ is a noncancellative product and $k=\ell$ otherwise. This is not a homomorphism of semirings ${ }^{\dagger}$, since $a+(-a)=\mathbb{O}_{W}$ whereas $v(-a)=v(a)$, and thus

$$
\psi_{\ell}(a+(-a))=\psi_{\ell}\left(\mathbb{O}_{W}\right)=\mathbb{O}_{R} \neq{ }^{[2 \ell]} a=\psi_{\ell}(a)+\psi_{\ell}(a)=\psi_{\ell}(a)+\psi_{\ell}(-a) .
$$

But this is exactly where the layered theory acts more categorically than the the max-plus theory.

Proposition 6.5. Suppose $W$ is an integral domain with valuation $v$, and

$$
\psi_{\ell}: \mathcal{M} \rightarrow \mathscr{R}(L, \mathcal{G})_{\mathfrak{a}},
$$

is the map just described. If $\sum_{i} a_{i}=\mathbb{O}_{W}$ with each $a_{i}$ in $W^{\times}$, then $s\left(\sum_{i} \psi_{\ell}\left(a_{i}\right)\right) \geq 2$.

Proof. This is really a reformulation of a standard, elementary fact in valuation theory, in which we recall that $v\left(\mathbb{O}_{W}\right)$ is undefined. It is well-known that if $\sum_{i} a_{i}=\mathbb{O}_{W}$ then there exist $i_{1}, i_{2}, \ldots$ such that $v\left(a_{i_{1}}\right)=v\left(a_{i_{2}}\right)=\ldots$ which dominate all other $v\left(a_{i}\right)$, since if a single $v\left(a_{i_{1}}\right)$ dominated, we would have $\mathbb{D}_{W}=v\left(\sum_{i} a_{i}\right)=v\left(a_{i_{1}}\right)$, a contradiction. Hence,

$$
s\left(\sum_{i} \psi_{\ell}\left(a_{i}\right)\right)=s\left(\psi_{\ell}\left(a_{i_{1}}\right)\right)+s\left(\psi_{\ell}\left(a_{i_{2}}\right)\right)+\cdots \geq 1+1+\cdots \geq 2 .
$$

Thus, we see that the $L$-tropicalization functor explains the importance of the "surpassing $L$-relation."

6.1.2. The role of Kapranov's Lemma. We are ready to extend the considerations of [19, §8.1]. Since Puiseux series play such an important role in tropical geometry, let us understand them in terms of layers.

Remark 6.6. We start with a triple $(F, \mathcal{G}, v)$, where $F$ for example may be the algebra of Puiseux series, $\mathcal{G}$ an ordered monoid, and $v: F \rightarrow \mathcal{G}$.

Take the layered semiring ${ }^{\dagger} R:=\mathscr{R}(L, \mathcal{G})$. Define a Kapranov map to be a $\{0,1\}$-supervaluation satisfying the property:

$$
\tilde{v}(a)+\tilde{v}(b) \underset{L}{\models} \tilde{v}(a+b) .
$$

This is the analog of the iq-supervaluation in [16. Definition 11.12]. By Proposition 6.5, we see that the Kapranov map sends any root of $f$ to a corner root of $\tilde{v}(f)$. This general framework of Kapranov's lemma encompasses tropicalizations of finite Puiseux series introduced in [17] and [19]. 


\section{LAYERED SUPERVALUATIONS AND TRANSMISSIONS: AN ALTERNATIVE APPROACH TO MORPHISMS}

In this section we delve deeper into the nature of morphisms, towards what would be the "correct" general definition in the category of layered semirings ${ }^{\dagger}$, paralleling the general theory of m-valuations given in [17. The outcome is somewhat technical, but enables us to define a functor from the functions in the algebraic world to the category of layered function semirings ${ }^{\dagger}$, and indicates that Payne's methods 27] should also be applicable in the layered theory.

In Corollary 7.11, we will see that this approach reduces to Section 6 in many cases.

Since valuations play such an important role, we would like to extend our definition of morphism to include all maps preserving valuations. This route leads us to a layered version of supervaluations and transmissions. See [16], [18], 20] for further details in the supertropical case.

Definition 7.1. An L-layered supervaluation on a ring $W$, with respect to a semiring $L$, is a map $\tilde{v}: W \rightarrow R$ from $W$ to an L-layered semiring $R$ satisfying the following properties.

$$
\begin{aligned}
& \text { LV1 }: \tilde{v}\left(\mathbb{1}_{W}\right)=\mathbb{1}_{R}, \\
& \text { LV2 }: \forall a, b \in R: \tilde{v}(a b)=\tilde{v}(a) \tilde{v}(b), \\
& \text { LV3 }: \forall a, b \in R: \tilde{v}(a+b) \leq_{\nu} \tilde{v}(a)+\tilde{v}(b), \\
& \text { LV4 }: \tilde{v}\left(\mathbb{O}_{W}\right)=\mathbb{0}_{R} .
\end{aligned}
$$

$A\{0,1\}$-supervaluation on a ring $W$ is an L-layered supervaluation $\tilde{v}: W \rightarrow R$ such that $\tilde{v}(W) \subseteq$ $R_{0} \cup R_{1}$.

An L-layered supervaluation ${ }^{\dagger}$ on an integral domain $W$, with respect to a semiring ${ }^{\dagger} L$, is a map $\tilde{v}: W^{\times} \rightarrow R$ from $W^{\times}:=W \backslash\left\{\mathbb{O}_{W}\right\}$ to an L-layered pre-domain ${ }^{\dagger} R$ with the following properties.

$$
\begin{aligned}
& \mathrm{LV}^{\dagger}: \tilde{v}\left(\mathbb{1}_{W}\right)=\mathbb{1}_{R}, \\
& \mathrm{LV}^{\dagger}: \forall a, b \in R: \tilde{v}(a b)=\tilde{v}(a) \tilde{v}(b), \\
& \mathrm{LV}^{\dagger}: \forall a, b \in R: \tilde{v}(a+b) \leq_{\nu} \tilde{v}(a)+\tilde{v}(b) .
\end{aligned}
$$

To encompass the results of [16] and 18, instead of using layered homomorphisms for our morphisms, we need to consider a "transmissive" property analogous to the one given in [18, Definition 4.3].

Definition 7.2. If $\tilde{v}: W \rightarrow R$ and $\widetilde{w}: W \rightarrow R^{\prime}$ are L-layered supervaluations, where $R$ has sorting map $s: R \rightarrow L$ and $R^{\prime}$ has sorting map $s^{\prime}: R^{\prime} \rightarrow L$, we say that $\tilde{v}$ dominates $\widetilde{w}$ if the following properties hold for any $a, b \in W$ :

$$
\begin{array}{ll}
\text { D1. } & \tilde{v}(a)=\tilde{v}(b) \Rightarrow \widetilde{w}(a)=\widetilde{w}(b), \\
\text { D2. } & \tilde{v}(a) \leq_{\nu} \tilde{v}(b) \Rightarrow \widetilde{w}(a) \leq_{\nu} \widetilde{w}(b), \\
\text { D3. } & \tilde{v}(a) \in R_{0} \Rightarrow \widetilde{w}(a) \in R_{0}^{\prime}, \\
\text { D4. } & s(\tilde{v}(a)) \leq s^{\prime}(\widetilde{w}(a)) \quad \text { whenever } \widetilde{w}(a) \notin R_{0}^{\prime} .
\end{array}
$$

(We omit D3 and the condition in D4 for layered supervaluations ${ }^{\dagger}$, since we do not need to bother with the 0 layer.)

Definition 7.3. For L-layered domains ${ }^{\dagger} R$ and $R^{\prime}$ and $\mathcal{M} \subset R$, a map $\alpha: \mathcal{M} \rightarrow R^{\prime}$ is $\nu$-preserving if

$$
a \leq_{\nu} b \quad \text { implies } \quad \alpha(a) \leq_{\nu} \alpha(b)
$$

for all $a, b \in R$.

Lemma 7.4. For any $\nu$-preserving map $\alpha$, if $a \cong{ }_{\nu} b$, then $\alpha(a) \cong{ }_{\nu} \alpha(b)$ for $a, b \in R$.

Proof. $a \cong_{\nu} b$ implies $\alpha(a) \leq_{\nu} \alpha(b)$ and likewise $\alpha(b) \leq_{\nu} \alpha(a)$, so $\alpha(a) \cong_{\nu} \alpha(b)$.

Lemma 7.5. Let $\tilde{v}: W \rightarrow R$ and $\widetilde{w}: W \rightarrow R^{\prime}$ be L-layered supervaluations. If $\tilde{v}$ dominates $\widetilde{w}$, then there exists a unique $\nu$-preserving map $\alpha_{\widetilde{w}, \tilde{v}}: \tilde{v}(W) \rightarrow R^{\prime}$ with $\widetilde{w}=\alpha_{\widetilde{w}, \tilde{v}} \circ \tilde{v}$.

Proof. By D1 we have a well-defined map $\alpha_{\widetilde{w}, \tilde{v}}: \tilde{v}(W) \rightarrow \widetilde{w}(W)$ given by $\alpha_{\widetilde{w}, \tilde{v}}(\tilde{v}(a))=\widetilde{w}(a)$ for all $a \in W$. Furthermore, if $\tilde{v}(a) \leq_{\nu} \tilde{v}(b)$, then D2 implies $\widetilde{w}(a) \leq_{\nu} \widetilde{w}(b)$, so $\alpha_{\widetilde{w}, \tilde{v}}$ is $\nu$-preserving. 
Definition 7.6. For layered semirings $R$ and $R^{\prime}$, a transmission from $R$ to $R^{\prime}$ is a $\nu$-preserving map $\alpha: \mathcal{M} \rightarrow R^{\prime}$, with $\mathcal{M}$ a multiplicative submonoid of $R$, satisfying the following axioms:

$$
\begin{array}{lll}
\text { TM1 : } & \alpha\left(\mathbb{1}_{R}\right)=\mathbb{1}_{R^{\prime}}, & \\
\text { TM2 : } & \alpha(a b)=\alpha(a) \alpha(b), & \forall a, b \in R, \\
\text { TM3 : } & \alpha(a+b) \cong_{\nu} \alpha(a)+\alpha(b), & \text { whenever } a, b, a+b \in \mathcal{M} .
\end{array}
$$

Axioms TM1 and TM2 imply that $\alpha$ is a monoid homomorphism, which we denote as $\alpha:(R, \mathcal{M}) \rightarrow R^{\prime}$ to emphasize that $\mathcal{M}$ is a submonoid of $R$. We write $\mathcal{M}_{\ell}$ for $R_{\ell} \cap \mathcal{M}$. $A\{0,1\}$-transmission from $R$ to $R^{\prime}$ is a transmission $\alpha:(R, \mathcal{M}) \rightarrow R^{\prime}$ for which $\alpha\left(\mathcal{M}_{1}\right) \subseteq R_{1}^{\prime} \cup R_{0}^{\prime}$.

Lemma 7.7. Axiom TM3 is equivalent to the map $\alpha$ being $\nu$-preserving.

Proof. $(\Rightarrow)$ If $a \leq_{\nu} b$, then

$$
\alpha(b) \cong_{\nu} \alpha(a+b) \cong_{\nu} \alpha(a)+\alpha(b),
$$

implying $\alpha(a) \leq_{\nu} \alpha(b)$.

$(\Leftarrow)$ We may assume that $a \leq_{\nu} b$, implying $a+b \cong_{\nu} b$. Then $\alpha(a) \leq_{\nu} \alpha(b)$, so

$$
\alpha(a)+\alpha(b) \cong_{\nu} \alpha(b) \cong_{\nu} \alpha(a+b) .
$$

Note that the condition of the lemma does not refer explicitly to calculating sums in $\mathcal{M}$, so we can study transmissions without worrying about addition on $\mathcal{M}$.

Theorem 7.8. Let $\tilde{v}: W \rightarrow R$ be an L-layered supervaluation and $\widetilde{w}: W \rightarrow R^{\prime}$ an L-layered supervaluation dominated by $\tilde{v}$. The map $\alpha:=\alpha_{\widetilde{w}, \tilde{v}}:(R, \tilde{v}(W)) \rightarrow R^{\prime}$ is a transmission from $R$ to $R^{\prime}$.

Conversely, assume that $\tilde{v}: W \rightarrow R$ is an L-layered supervaluation and $\alpha: \tilde{v}(W) \rightarrow R^{\prime}$ is a transmission from $R$ to an L-layered semiring ${ }^{\dagger} R^{\prime}$. Then $\alpha \circ \tilde{v}: W \rightarrow R^{\prime}$ is an L-layered supervaluation dominated by $\tilde{v}$.

Proof. TM1 and TM2 are obtained from the construction of $\alpha_{\widetilde{w}, \tilde{v}}$ in the proof of Lemma 7.5. Now assume that $a \leq_{\nu} b$, so $\tilde{v}(a) \leq_{\nu} \tilde{v}(b)$, and thus $\tilde{v}(a)+\tilde{v}(b) \cong_{\nu} \tilde{v}(b)$. But $\widetilde{w}(a) \leq_{\nu} \widetilde{w}(b)$ by D2, so

$$
\alpha(\tilde{v}(a))+\alpha(\tilde{v}(b))=\widetilde{w}(a)+\widetilde{w}(b) \cong_{\nu} \widetilde{w}(b)=\alpha(\tilde{v}(b)) \cong_{\nu} \alpha(\tilde{v}(a)+\tilde{v}(b)) .
$$

This is TM3.

For the reverse direction, let $\widetilde{w}:=\alpha \circ \tilde{v}$, Clearly $\widetilde{w}$ inherits the properties LV1-LV3 from $\tilde{v}$, since $\alpha$ satisfies TM1-TM3.

Corollary 7.9. Every transmission of Theorem 7.8 is $\nu$-preserving.

Proof. $\alpha$ is the map of Lemma 7.5 so is $\nu$-preserving.

It is evident that every semiring ${ }^{\dagger}$ homomorphism from $R$ to $R^{\prime}$ is a transmission, but there exist transmissions that are not semiring ${ }^{\dagger}$ homomorphisms; cf. [16, §9]. Nevertheless, we do get semiring ${ }^{\dagger}$ homomorphisms in the following basic case. We say that the transmission $\alpha$ is homomorphic if it satisfies the condition

$$
\alpha(a+b)=\alpha(a)+\alpha(b)
$$

whenever $a, b, a+b \in \mathcal{M}$.

Every homomorphic transmission satisfying $\mathcal{M}=R$ is a layered homomorphism, by definition. We say that a $\nu$-preserving map $\alpha$ is strictly $\nu$-preserving if $a<_{\nu} b$ implies that either $\alpha(a) \in R_{0}^{\prime}$ or $\alpha(a)<\nu^{\prime} \alpha(b)$.

Theorem 7.10. Let $\tilde{v}: W \rightarrow R$ be an $\{0,1\}$-layered supervaluation and $\widetilde{w}: W \rightarrow R^{\prime}$ an $\{0,1\}$ layered supervaluation dominated by $\tilde{v}$. Then the $\{0,1\}$-transmission $\alpha:=\alpha_{\widetilde{w}, \tilde{v}}:(R, \tilde{v}(W)) \rightarrow R^{\prime}$ is homomorphic, iff it is strictly $\nu$-preserving. 
Proof. $(\Rightarrow)$ Follows from Corollary 7.9 .

$(\Leftarrow)$ We need to check (7.1). If $a<_{\nu} b$, then $\alpha(a+b)=\alpha(b)$, so (7.1) holds iff $\alpha(a)<_{\nu} \alpha(b)$ or $\alpha(a) \in R_{0}$. The symmetric argument holds when $b<_{\nu} a$. Finally, if $a \cong_{\nu} b$, with $a \in R_{0}$, then $\alpha(a) \in R_{0}$, with $\alpha(a) \cong{ }_{\nu} \alpha(b)$, so

$$
\alpha(a+b)=\alpha(b)=\alpha(a)+\alpha(b) .
$$

Likewise for $b \in R_{0}$, so we may assume that $a, b \in R_{1}$. Then $a+b \in R_{2}$, so there is nothing to check.

Corollary 7.11. Suppose $\tilde{v}: W \rightarrow R$ is an $\{0,1\}$-layered supervaluation such that $\tilde{v}(W)$ strictly generates $R$, and $\widetilde{w}: W \rightarrow R^{\prime}$ is an $\{0,1\}$-layered supervaluation dominated by $\tilde{v}$. Then the $\{0,1\}$-transmission $\alpha:=\alpha_{\widetilde{w}, \tilde{v}}:(R, \tilde{v}(W)) \rightarrow R^{\prime}$ extends to a layered homomorphism from $R$ to $R^{\prime}$, iff $\alpha$ is strictly $\nu$ preserving.

In particular, when $R$ is uniform, every $\{0,1\}$-transmission yields a layered homomorphism.

Remark 7.12. Since every transmission is a monoid homomorphism, we have a subcategory L-STROP of the category of monoids and monoid homomorphisms, whose objects are layered semirings $\left(R, L, s,\left(\nu_{m, \ell}\right)\right)$, and whose morphisms are the $\{0,1\}$-transmissions. Explicitly, $\{0,1\}$-transmissions from $R$ to $R^{\prime}$ and from $R^{\prime}$ to $R^{\prime \prime}$ are described respectively as transmissions $\alpha:(R, \mathcal{M}) \rightarrow R^{\prime}$ for which $\alpha\left(\mathcal{M}_{1}\right) \subseteq R_{1}^{\prime} \cup R_{0}^{\prime}$ and $\alpha^{\prime}:\left(R^{\prime}, \mathcal{M}^{\prime}\right) \rightarrow R^{\prime}$ for which $\alpha^{\prime}\left(\mathcal{M}_{1}^{\prime}\right) \subseteq R_{1}^{\prime \prime} \cup R_{0}^{\prime \prime}$. Their composition can be defined as $\alpha^{\prime} \circ \alpha$ whenever $\alpha(\mathcal{M}) \subseteq \mathcal{M}_{1}^{\prime}$.

This category closely resembles the category STROP of [18] (but with the subtle difference indicated in Remark 4.6), and encompasses the category from 86.1 .

\section{APPENDIX: LAYERED MONOIDS}

At times we do not want additivity at the 0 level, since the vagaries of cancellation complicate the statements and proofs some of the theorems. But then we must give up addition between $R_{0}$ and other levels. At this generality, our next structure is not quite a semiring ${ }^{\dagger}$, since distributivity does not hold at the 0-layer, but we copy what we can from Definition 4.1 .

Definition 8.1. Suppose $(L, \geq)$ is a directed, partially pre-ordered semiring ${ }^{\dagger}$. An L-layered monoid

$$
R:=\left(R, L, s,\left(\nu_{m, \ell}\right)\right),
$$

is a multiplicative monoid $R$ which is a disjoint union of subsets $R_{\ell}, \ell \in L$, together with addition defined on $R_{0}$ and on $R_{>0}:=\dot{\bigcup}_{\ell>0} R_{\ell}$ such that

$$
R=\bigcup_{\ell \in L} R_{\ell},
$$

together with a family of sort transition maps

$$
\nu_{m, \ell}: R_{\ell} \rightarrow R_{m}, \quad \forall m \geq \ell>0,
$$

such that

$$
\nu_{\ell, \ell}=\operatorname{id}_{R_{\ell}}
$$

for every $\ell \in L$, and

$$
\nu_{m, \ell} \circ \nu_{\ell, k}=\nu_{m, k}, \quad \forall m \geq \ell \geq k,
$$

whenever both sides are defined. We also require the axioms $A 1-A 4$, and $B$, given presently, to be satisfied.

We define $R_{\infty}$ to be the direct limit of the $R_{\ell}, \ell>0$, together with a map $\nu: R_{\ell} \rightarrow R_{\infty}$, which extends to a map $\nu: R \rightarrow R_{\infty}$. We write $a^{\nu}$ for $\nu(a)$.

We write $a \cong{ }_{\nu} b$ for $b \in R_{\ell}$, whenever $\nu(a)=\nu(b)$. (For $k, \ell>0$ this means $\nu_{m, k}(a)=\nu_{m, \ell}(b)$ in $R_{m}$ for some $m \geq k, \ell$. The notation is used generically, as before.) Similarly, we write $a \leq_{\nu} b$ if $a \cong_{\nu} b$ or $\nu_{m, k}(a)+\nu_{m, \ell}(b)=\nu_{m, \ell}(b)$ in $R_{m}$ for some $m \geq k, \ell$.

The axioms are as follows: 
A1. $\mathbb{1}_{R} \in R_{1}$.

A2. If $a \in R_{k}$ and $b \in R_{\ell}$, then $a b \in m$ where $m \geq k \ell$ or $m=0$.

$\mathrm{A} 2^{\prime}$. If $a \in R_{0}$ or $b \in R_{0}$, then $a b \in R_{0}$.

A3. The product in $R$ is compatible with sort transition maps: Suppose $a \in R_{\ell}, b \in R_{\ell}^{\prime}$, with $m \geq \ell$ and $m^{\prime} \geq \ell^{\prime}$.

If $a b \in R_{\ell^{\prime \prime}}$ for $\ell^{\prime \prime} \geq \ell^{\prime} \ell$, then $\nu_{m, \ell}(a) \cdot \nu_{m^{\prime}, \ell^{\prime}}(b)=\nu_{m^{\prime \prime}, \ell^{\prime \prime}}(a b)$ for some $m^{\prime \prime} \geq m m^{\prime}$.

A4. $\nu_{\ell, k}(a)+\nu_{\ell^{\prime}, k}(a)=\nu_{\ell+\ell^{\prime}, k}(a)$ for all $a \in R_{k}$ and all $\ell, \ell^{\prime} \geq k$.

A5. If $a \in R_{k}, b \in R_{\ell}$, and $c=a+b \in R_{k^{\prime}}$, then

$$
\nu_{m, k^{\prime}}(c)=\nu_{m, k}(a)+\nu_{m, \ell}(b)
$$

for each $m \geq k+\ell$.

A6. $R_{>0}$ is an additive semigroup and $R_{0}$ is an $R_{>0}$-module, in the sense that $R_{0}$ is an additive semigroup together with a multiplication $R_{0} \times R_{>0} \rightarrow R_{0}$ satisfying distributivity and associativity whenever defined.

B. (Supertropicality) Suppose $a \in R_{k}, b \in R_{\ell}$, and $a \cong_{\nu} b$. Then $a+b \in R_{k+\ell}$ with $a+b \cong_{\nu} a$.

The sorting map $s: R \rightarrow L$, is a map that sends every element $a \in R_{\ell}$ to its sort $\ell$.

Example 8.2. Suppose $R$ is a layered pre-domain ${ }^{\dagger}$, and define formally $R_{0}$ to be another copy of $R$ with 0 adjoined in the natural way, where we write $e_{0}$ for its multiplicative unit. Then $R \cup R_{0}$ is naturally a layered monoid, where we define $\left(e_{0} a\right) b:=e_{0}(a b)$ and $e_{0} a+e_{0} b=e_{0}(a+b)$.

Remark 8.3. What we are lacking for obtaining a semiring is the definition of $a+b$ for $a \cong_{\nu} b$ with $a \in R_{0}$ and $b \in R_{\ell}$ for $\ell>0$. The natural guess might be to define $a+b=b$ in this case, but this could ruin distributivity. If there happens to be $c \in R$ such that $b c \in R_{0}$, then we would have $(a+b) c=b c$, which does not necessarily equal $a c+b c$.

In this generality, we also need a more intricate definition of morphism.

Definition 8.4. A layered morphism of tangibly generated L-layered monoids is a map

$$
\Phi:=(\varphi, \rho):\left(R, L, s,\left(\nu_{m, \ell}\right)\right) \rightarrow\left(R^{\prime}, L^{\prime}, s^{\prime},\left(\nu_{m^{\prime}, \ell^{\prime}}^{\prime}\right)\right)
$$

such that $\rho: L \rightarrow L^{\prime}$ is a semiring homomorphism, together with a multiplicative monoid homomorphism $\varphi: R \rightarrow R^{\prime}$ that also preserves addition on $R_{>0}$ in the sense that $\varphi(a+b)=\varphi(a)+\varphi(b)$ for all $a, b$ in $R_{>0}$, and which also satisfies the following properties:

M1. If $\varphi(a) \notin R_{0}^{\prime}$, then $\mathrm{s}^{\prime}(\varphi(a)) \geq \rho(\mathrm{s}(a))$ or $\mathrm{s}^{\prime}(\varphi(a))=0$.

M2. $\varphi\left(a^{\nu}\right) \cong_{\nu} \varphi(a)$.

M3. If $a \cong_{\nu} b$, then $\varphi(a) \cong_{\nu} \varphi(b)$.

The ensuing category closely resembles the category $\mathrm{STROP}_{m}$ of $[20]$.

\subsection{Weakening the structure of $L$ and $R$.}

Note 8.5. To generalize the notion "supertropical semiring" from the standard supertropical theory, we could weaken Axiom A2 to:

wA2. If $a \in R_{k}$ and $b \in R_{\ell}$, then $a b \in R_{m}$ for some $m \geq k \ell$. 
Now we have to modify Axiom A3 to make it compatible; i.e., multiplication commutes with the sort transition maps. Technically, this says:

$$
\begin{aligned}
& \text { wA3. If } a \in R_{k} \text { and } a^{\prime} \in R_{k^{\prime}} \text {, with } a a^{\prime} \in R_{k^{\prime \prime}} \text { and } \nu_{\ell, k}(a) \cdot \nu_{\ell^{\prime}, k^{\prime}}\left(a^{\prime}\right) \in \\
& R_{\ell^{\prime \prime}} \text { and } \nu_{m, \ell}(a) \cdot \nu_{m^{\prime}, \ell^{\prime}}\left(a^{\prime \prime}\right) \in R_{m^{\prime \prime}}, \text { for } m \geq \ell, m^{\prime} \geq \ell^{\prime} \text {, and } \\
& m^{\prime \prime} \geq m m^{\prime} \text {, then } \\
& \nu_{q, \ell^{\prime \prime}}\left(a a^{\prime}\right)=\nu_{q, m^{\prime \prime}}\left(\nu_{m, \ell}(a) \cdot \nu_{m^{\prime}, \ell^{\prime}}\left(a^{\prime}\right)\right) \text { for all } q \geq \ell^{\prime \prime}, m^{\prime \prime} \text {. }
\end{aligned}
$$

This weakening is of arithmetic interest, since we now have a version of the theory without requiring a zero layer.

Remark 8.6. We do not need $L$ to be a semiring ${ }^{\dagger}$, but merely a directed, partially pre-ordered multiplicative monoid (without addition). This material yields an intriguing parallel between the layered monoid $R$ and the sorting set $L$ (since any ordered monoid becomes a semiring ${ }^{\dagger}$ when addition is taken to be the maximum), and may provide guidance for future research.

Since $L$ now is only assumed to be a multiplicative monoid, we need to remove references to addition in L. Thus, we need a formal "doubling function" $\ell \mapsto 2 \ell$ on $L$, eliminate Axiom A 4 , and weaken Axiom $B$ to:

wB. (weak supertropicality) If $a \in R_{k}$ and $b \in R_{\ell}$ with $a \cong_{\nu} b$, then $a+b \in R_{m}$ for some $m \geq k, \ell, \min \{2 k, 2 \ell\}$ with $a+b \cong_{\nu} b$.

\section{REFERENCES}

[1] M. Akian, R. Bapat, and S. Gaubert. Max-plus algebra, In: Hogben, L., Brualdi, R., Greenbaum, A., Mathias, R. (eds.) Handbook of Linear Algebra. Chapman and Hall, London, 2006.

[2] M. Akian, S. Gaubert, and A. Guterman, Linear independence over tropical semirings and beyond. In Tropical and Idempotent Mathematics, G.L. Litvinov and S.N. Sergeev, (eds.), Contemp. Math., 495:1-38, 2009.

[3] V. Berkovich, Spectral theory and analytic geometry over non-Archimedean fields, Mathematical surveys and monographs 33, Amer. Math. Soc., 1990

[4] A. Connes and C. Consani, Characteristic 1, entropy, and the absolute point. Preprint at arXiv:math.0911.3537, 2009.

[5] A.A. Costa, Sur la thêorie générale des demi-anneaux, Publ. Math. Decebren 10 (1963), 14-29.

[6] G. Cortinas, C. Haesemeyer, M. Walker, and C. Weibel, Toric varieties, monoid schemes, and descent. (preprint, 2010)

[7] A. Dickenstein, E. M. Feichtner, and B. Sturmfels, Tropical discriminants. J. Amer. Math. Soc, (20):1111-1133, 2007.

[8] M. Dubey, Some results on semimodules analogous to module theory. Doctoral dissertation. University of Delhi.

[9] M. Einsiedler, M. Kapranov, and D. Lind, Non-Archimedean amoebas and tropical varieties. J. Reine Angew. Math., 601:139-157, 2006.

[10] J. Golan, The theory of semirings with applications in mathematics and theoretical computer science, vol 54. Longman Sci \& Tech., 1992.

[11] D.K. Harrison and M.A. Vitulli, V-valuations of a commutative ring I, J. Algebra 126 (1989), 264-292.

[12] R. Hartshorne, Algebraic Geometry. Number 52 in Graduate Texts in Mathematics. Springer, 1977.

[13] H.C. Hutchins and H.J. Weinert, Homomorphisms and kernels of semifields Periodica Mathematica Hungaria, 21(2):113-152, 1990.

[14] I. Itenberg, G. Mikhalkin, and E. Shustin, Tropical algebraic geometry, Oberwolfach seminars, vol. 35. Birkhauser, 2007.

[15] Z. Izhakian, Tropical arithmetic and matrix algebra. Comm. in Algebra, 37(4):1445-1468, 2009.

[16] Z. Izhakian, M. Knebusch, and L. Rowen, Supertropical semirings and supervaluations. J. Pure and Appl. Alg., 215(10): 2431-2463, 2011.

[17] Z. Izhakian, M. Knebusch, and L. Rowen, Layered tropical mathematics. Preprint at arXiv:0912.1398, 2011

[18] Z. Izhakian, M. Knebusch, and L. Rowen, Dominance and transmissions in supertropical valuation theory. Comm. in Algebra, to appear. (Preprint at arXiv:1102.1520)

[19] Z. Izhakian, M. Knebusch, and L. Rowen, Categorical notions of layered tropical algebra and geometry, preprint, 2011.

[20] Z. Izhakian, M. Knebusch, and L. Rowen, Supertropical monoids: Basics, canonical factorization, and lifting ghosts to tangibles, preprint at arXiv:1108.1880, 2011.

[21] Z. Izhakian and L. Rowen, Supertropical algebra, Adv. in Math. 324(8):1860-1886, 2010.

[22] Z. Izhakian and L. Rowen, Supertropical matrix algebra. Israel J. Math. 182(1):383-424, 2011.

[23] N. Jacobson, Basic Algebra II. Freeman, 1980.

[24] G. Litvinov, The Maslov dequantization, idempotent and tropical mathematics: a very brief introduction. J. of Math. Sciences, 140(3): 426-444, 2007.

[25] D. Maclagan and B. Sturmfels, Tropical Geometry. Preprint, 2009.

[26] A. Patchkoria, On derived functors of semimodule-valued functors, Proc. A. Razmadze Math. Inst. (in Russian) 83:60$75,1986$. 
[27] S. Payne, Fibers of tropicalization, Math Z. 262 (2): 301-311, 2009.

[28] S. Payne, Analytification is the limit of all tropicalizations, Preprint at arXiv: 0806.1916v3 [math.AG], 2009.

[29] L. Rowen, Graduate algebra: Commutative view. Graduate Studies in Mathematics 73, 2006. American Mathematical Society 91, 2006.

[30] L. Rowen, Graduate algebra: Noncommutative view. Graduate Studies in Mathematics 73, 2006. American Mathematical Society, 2006.

[31] D. Zhang, The M-valuation spectrum of a commutative ring, Comm. in Algebra 30 (2002), $2883-2896$.

Department of Mathematics, Bar-Ilan University, Ramat-Gan 52900, Israel

E-mail address: zzur@math.biu.ac.il

Department of Mathematics, University of Regensburg, Regensburg, Germany

E-mail address: manfred.knebusch@mathematik.uni-regensburg.de

Department of Mathematics, Bar-Ilan University, Ramat-Gan 52900, Israel

E-mail address: rowen@macs.biu.ac.il 